\title{
Campos de caminhos em variedades topológicas
}

\author{
Paulo Augusto Ribeiro
}

DisSERTAÇÃo APRESENTADA

$\mathrm{AO}$

Instituto De Matemática e Estatística

DA

Universidade De SÃo PaUlo

PARA

OBTENÇÃO DO TÍTULO

$\mathrm{DE}$

Mestre em CiÊnCIAS

Programa: Matemática

Orientadora: Profa. Dra. Lucília Daruiz Borsari

Durante o desenvolvimento deste trabalho o autor recebeu auxílio financeiro da

$$
\text { CAPES/CNPq }
$$

São Paulo, dezembro de 2010 


\section{Campos de caminhos em variedades topológicas}

Esta versão definitiva da dissertação contém as correções e alterações sugeridas pela Comissão Julgadora durante a defesa realizada por Paulo Augusto Ribeiro em 13/12/2010.

Comissão Julgadora:

- Profa. Dra. Lucília Daruiz Borsari (orientadora) - IME-USP

- Profa. Dra. Fernanda S. P. Cardona - IME-USP

- Prof. Dr. Oziride Manzoli Neto - ICMC-USP 


\section{Dedicatória}

Dedico este trabalho aos meus familiares, amigos e professores que me incentivaram e ajudaram durante todo este período. 


\section{Resumo}

Esta dissertação expõe o estudo realizado sobre o artigo de R. Brown, citado na bibliografia, e sobre os conceitos necessários para a compreensão deste material.

Entre os principais conceitos e resultados preliminares discutidos, podemos citar: topologia de espaços de funções, teoria de homotopia, espaços compactos ANR, característica de Euler de um compacto ANR, teorema de Lefschetz, espaços fibrados, e campos de caminhos.

Os principais resultados discutidos na dissertação são os teoremas centrais do artigo de Brown: toda n-variedade topológica compacta admite um campo de caminhos com no máximo uma singularidade; e, uma n-variedade topológica compacta orientável admite um campo de caminhos sem singularidades se, e somente se, sua característica de Euler é zero. Discutimos também, suas respectivas consequências em teoria de ponto fixo.

Palavras-chave: variedades topológicas, campos de caminhos, característica de Euler. 


\section{Abstract}

This essay has the purpose of exposing the studies on the paper by R. Brown, quoted on the references, and on the concepts necessary to the comprehension of it.

Among the main concepts and preliminary results discussed, we can cite: topology of function spaces, homotopy theory, ANR compact spaces, Euler characteristic of a compact ANR, Lefschetz theorem, fiber spaces, and field paths.

The main results discussed in the text are the central theorems presented on Brown's paper: every compact topological n-manifold admits a path field with at most one singularity, and a compact orientable topological n-manifold M admits a nonsingular path field if and only if the Euler characteristic of $\mathrm{M}$ is zero. We also discussed their consequences on fixed point theory.

Keywords: topological manifolds, path fields, Euler characteristic. 


\section{Sumário}

Introdução

1 Preliminares em topologia geral $\quad 3$

1.1 Definições e resultados iniciais . . . . . . . . . . . . . . . . . . 3

1.2 Topologia de espaços de funções . . . . . . . . . . . . . . . . . 5

2 Variedades Topológicas e o Teorema da Dominação 11

2.1 Simplexos e complexos simpliciais . . . . . . . . . . . . . . 11

2.2 Coberturas estrela finitas e nervo de uma cobertura . . . . . . . . . . 12

2.3 Dimensão de um espaço métrico . . . . . . . . . . . . . . . . . . . . . 14

2.4 Retratos e extensões . . . . . . . . . . . . . . . . . . 15

2.5 Homotopia e extensão de homotopias . . . . . . . . . . . . . . 16

2.6 Teorema da Dominação . . . . . . . . . . . . . . . . . . . . . . . 17

2.7 Variedades topológicas . . . . . . . . . . . . . . . . . 20

3 Espaços fibrados $\quad 23$

3.1 O conceito de espaço fibrado . . . . . . . . . . . . . . 23

3.2 Par fibrado . . . . . . . . . . . . . . . . . . . 30

3.3 Fibrado vetorial generalizado e principais resultados . . . . . . . . . 31

4 Campos de caminhos em variedades topológicas 41

4.1 Campos de caminhos com apenas uma singularidade . . . . . . . . . 41 
4.2 Número de Lefschetz e característica de Euler . . . . . . . . . . . . 46

4.3 Índice de ponto fixo . . . . . . . . . . . . . . . . . . . . 48

4.4 Campos de caminhos sem singularidades . . . . . . . . . . . 51 


\section{Introdução}

Esta dissertação expõe o estudo realizado sobre o artigo de R. Brown, Path Fields on Manifolds [3], juntamente com os conceitos e resultados básicos necessários para a compreensão deste material. O objetivo do trabalho de Brown é generalizar para variedades topológicas um resultado conhecido em geometria diferencial válido para variedades diferenciáveis:

Uma variedade diferenciável compacta orientável admite um campo de vetores sem singularidades se, e somente se, sua característica de Euler é zero.

Após estabelecer a versão deste teorema para variedades topológicas, não necessariamente diferenciáveis, é apresentada a respectiva consequência em teoria de ponto fixo.

No trabalho entitulado A Path Space And The Stiefel-Whitney Classes [20], J. Nash introduz um subconjunto $T_{0}$ do espaço de caminhos de uma variedade topológica $M$, consistindo em caminhos $\omega$ tais que $\omega(t)=\omega(0)$ se, e somente se, $t=0$. Denotamos por $T$ a união de $T_{0}$ com os caminhos constantes em $M$, atribuímos a $T$ a topologia compacto-aberta e definimos uma projeção $q: T \rightarrow M$ por $q(\omega)=\omega(0)$; como veremos, segue das definições destes conjuntos que $(T, q, M)$ é um espaço fibrado no sentido de Hurewicz [16] e, no caso de $M$ ser uma variedade diferenciável, possui o mesmo tipo homotópico, como espaço fibrado, que o fibrado tangente de $M$, sendo portanto, uma generalização deste conceito para o caso não diferenciável. Por analogia a noção de campo de vetores, definimos um campo de caminhos como sendo uma seção em $(T, q, M)$.

A generalização do resultado acima é, portanto, enunciada da seguinte forma:

Uma variedade topológica compacta orientável admite um campo de caminhos sem singularidades se, e somente se, sua característica de Euler é zero.

A fim de estabelecer os conceitos necessários para demonstração deste resultado, iniciamos com uma discussão sobre topologia de espaços de funções, em particular, discutimos a topologia compacto-aberta, que nos fornece uma maneira bastante prática para lidarmos com espaços de funções, e portanto, com espaços de caminhos. 
Como mostraremos no primeiro capítulo, com algumas condições sobre os espaços $X$ e $T$, obtemos um homeomorfismo entre $Y^{X \times T}$ e $\left(Y^{X}\right)^{T}$.

O capítulo seguinte tem como principais objetivos demonstrar o Teorema da Dominação, segundo o qual uma classe bastante ampla de espaços topológicos, mais precisamenete os espaços compactos ANR, são dominados por poliedros; e ainda, estabelecer certas propriedades das variedades topológicas, inclusive o fato de que toda variedade topológica é um compacto ANR, e portanto, é dominada por um poliedro, fato que será fortemente utilizado no capítulo seguinte.

No terceiro capítulo discutimos sobre espaços fibrados, estabelecemos a equivalência deste conceito com a importante propriedade de levantamento de homotopias absoluta e apresentamos uma classe bastante ampla de espaço fibrados, definimos um $n$-fibrado vetorial generalizado ( $\mathrm{n}-\mathrm{fvg}$ ). Em seguida, iniciamos as demonstrações de uma série de resultados que concluirão na demonstração de que se $\mathcal{F}$ é um n-fvg sobre uma variedade topológica então existe uma seção de $\mathcal{F}$ com no máximo uma singularidade.

No capítulo final demonstramos que $(T, q, M)$ é um n-fvg, e obtemos uma versão próxima ao resultado buscado juntamente com a respectiva consequência em teoria de ponto fixo, concluímos que: Toda variedade topológica compacta admite um campo de caminhos com no máximo uma singularidade. Este resultado reduz nosso problema a uma questão local e mais simples de se manipular. Logo após, desenvolvemos os conceitos de Número de Lefschetz, Característica de Euler e Índice de Ponto Fixo que nos permitem concluir a demonstração desejada. Por fim, obtemos o corolário em teoria de ponto fixo: Se $M$ é uma variedade orientável compacta, então existe uma aplicação $f: M \rightarrow M$ sem pontos fixos e homotópica à identidade se, $e$ somente se, $\chi(M)=0$. 


\section{Capítulo 1}

\section{Preliminares em topologia geral}

\subsection{Definições e resultados iniciais}

Sejam $X$ um espaço topológico e $\mathcal{B}$ uma coleção de subconjuntos abertos de $X$, a coleção $\mathcal{B}$ é uma base de $X$ se todo aberto de $X$ pode ser escrito como a união de elementos de $\mathcal{B}$, e $\mathcal{B}$ é uma subbase de $X$ se, as interseções finitas $B_{1} \cap \ldots \cap B_{n}$, $B_{i} \in \mathcal{B}$, formam uma base de $X$. Consequentemente, a topologia de $X$ gerada por esta subbase é a menos fina na qual todos os elementos de $\mathcal{B}$ são abertos.

Uma coleção $\mathcal{U}=\left\{U_{\mu}\right\}_{\mu \in M}$ de subconjuntos de um espaço $X$ é uma cobertura de $X$ se

$$
X=\bigcup_{\mu \in M} U_{\mu}
$$

Se todos os conjuntos $U_{\mu}$ são abertos (fechados) em $X, \mathcal{U}$ é uma cobertura por abertos (fechados) de $X$. Se $M$ é um conjunto enumerável, $\mathcal{U}$ é uma cobertura enumerável. Uma cobertura $\mathcal{V}=\left\{V_{\nu}\right\}_{\nu \in N}$ é um refinamento de $\mathcal{U}$ se, para todo $\nu \in N$, existe $\mu \in M$ tal que $V_{\nu} \subset U_{\mu}$.

Uma cobertura $\mathcal{U}=\left\{U_{\mu}\right\}_{\mu \in M}$ de $X$ é localmente finita se, para todo $x \in X$, existe uma vizinhança $W$ de $x$, tal que $W \cap U_{\mu} \neq \emptyset$ apenas para um número finito de $\mu$ 's. Um espaço é paracompacto se toda cobertura por abertos deste espaço possui um refinamento aberto localmente finito.

Um espaço topológico $X$ é localmente compacto se todo ponto de $X$ possui um sistema fundamental de vizinhanças compactas.

Sejam $X$ um espaço topológico e $x, y$ elementos distintos de $X$.

1. $X$ é $T_{0}$ se existe um aberto que contém apenas um desses pontos;

2. $X$ é $T_{1}$ se existe uma vizinhança de $x$ que não contém $y$; 
3. $X$ é $T_{2}$, ou de Hausdorff, se existem vizinhanças $U$ de $x$ e $V$ de $y$ tais que $U \cap V=\emptyset$;

4. $X$ é $T_{3}$ se, para cada $x \in X$, e cada fechado $F \subset X$ tal que $x \notin F$, existem abertos $U$ contendo $x$ e $V$ contendo $F$ tais que $U \cap V=\emptyset$;

5. $X$ é $T_{4}$ se, para cada par de fechados $\mathrm{F}$ e $\mathrm{G}$ disjuntos, existem abertos disjuntos $U$ e $V$ contendo $F$ e $G$, respectivamente.

Temos, assim, as seguintes equivalências:

1. $X$ é $T_{0} \Leftrightarrow \overline{\{x\}} \neq \overline{\{y\}}$;

2. $X$ é $T_{1} \Leftrightarrow$ para cada $x \in X,\{x\}$ é fechado;

3. $X$ é Hausdorff $\Leftrightarrow$ para cada $x \in X$, a intersecção de todas as vizinhanças fechadas de $x$ é o conjunto $\{x\}$;

4. $X$ é $T_{3} \Leftrightarrow$ para cada $x \in X$ e para cada vizinhança aberta $W$ de $x$, existe uma vizinhança aberta $U$ de $x$ tal que $\bar{U} \subset W$;

5. $X$ é $T_{4} \Leftrightarrow$ para cada par de fechados $F$ e $G$ disjuntos, existe um aberto $U$ tal que $F \subset U \subset \bar{U} \subset X \backslash G$.

O espaço $X$ é regular se $X$ é $T_{3}$ e $T_{1}$; e normal se $X$ é $T_{4}$ e $T_{1}$.

Seja $I^{\omega}$ o cubo de Hilbert, ou seja, o conjunto de todas as sequências de números reais $\left(x_{1}, x_{2}, \ldots\right)$ tais que $\left|x_{i}\right|<1 / i$ para todo $i$, munido com a topologia induzia pela métrica

$$
d\left(\left(x_{1}, x_{2}, \ldots\right),\left(x_{1}^{\prime}, x_{2}^{\prime}, \ldots\right)\right)=\left(\sum_{i=1}^{\infty}\left(x_{i}-x_{i}^{\prime}\right)\right)^{1 / 2} .
$$

O cubo de Hilbert é um espaço métrico compacto, e possui base enumerável.

Proposição 1 (Teorema da metrização de Urysohn). Se X é um espaço topológico $T_{4}$, Hausdorff e com base enumerável, então existe uma função contínua $F: X \rightarrow I^{\omega}$ tal que $F$ é um homeomorfismo de $X$ na sua imagem $F(X)$.

Proposição 2 (Teorema da extensão de Tietze-Urysohn). Sejam X um espaço topológico $T_{4}$ e $M$ um subespaço fechado de $X$. Então toda função contínua definida em $M$ com valores em $[0,1]$ ou $\mathbb{R}$ pode ser estendida continuamente a $X$.

As demonstrações destes resultados podem ser encontradas em [18, p. 234] e [18, p. 286], respectivamente. 


\subsection{Topologia de espaços de funções}

Dados espaços topológicos $X, T$ e $Y$ e uma função $h$ de $X \times T$ em $Y$ que é contínua em $x \in X$ para cada $t \in T$ fixado, podemos associar à $h$ uma função $\theta(h)$ de $T$ em $Y^{X}$, onde $Y^{X}$ é o espaço das funções contínuas de $X$ em $Y$. A função $\theta(h)$ é dada por

$$
\theta(h)(t)=h_{t}, \quad \text { onde } \quad h_{t}(x)=h(x, t) .
$$

Para determinamos a continuidade de uma função $h$ específica dependemos da topologia dos espaços $X, T$ e $Y$, porém, para determinarmos a continuidade da função $\theta(h)$, dependemos da topologia do espaço $Y^{X}$. Desejamos atribuir a este espaço uma topologia tal que uma função $\theta(h)$ seja contínua precisamente quando a função $h$ for contínua.

Dados $K \subset X$ e $U \subset Y$ denotemos por $W(K, U)$ o subconjunto de $Y^{X}$ formado pelas funções contínuas que levam $K$ em $U$, ou seja,

$$
W(K, U)=\left\{f \in Y^{X} \mid f(K) \subset U\right\} .
$$

A coleção dos conjuntos $W(K, U)$ tais que $K$ é compacto em $X$ e $U$ é aberto em $Y$ é uma subbase de $Y^{X}$, a topologia gerada por esta subbase é a topologia compactoaberta. A menos que mencionado o contrário, $Y^{X}$ denotará o espaço topológico formado por este conjunto munido com a topologia compacto-aberta.

Os resultados a seguir foram adaptados de [14].

Proposição 3. Para espaços topológicos arbitrários $X, T$ e $Y$ temos que se $h$ é contínua, então $\theta(h)$ é contínua, ou seja, $\theta\left(Y^{X \times T}\right) \subset\left(Y^{X}\right)^{T}$.

Demonstração. Sejam $g=\theta(h): T \rightarrow Y^{X}$ e um aberto de $Y^{X}$ da forma $W=$ $W(K, U)$. Basta provar que $g^{-1}(W)$ é um aberto de $T$. Dado $t_{0} \in g^{-1}(W)$, temos que

$$
\left[g\left(t_{0}\right)\right](K) \subset U \quad \text { e } \quad\left[g\left(t_{0}\right)\right](K)=h\left(K \times t_{0}\right),
$$

$\log \mathrm{O}$

$$
h\left(K \times t_{0}\right) \subset U \quad \text { e } \quad K \times t_{0} \subset h^{-1}(U) .
$$

Como $h$ é contínua, temos que $h^{-1}(U)$ é um aberto de $X \times T$, portanto, $h^{-1}(U)$ é uma união de abertos da forma $A_{\mu} \times B_{\mu}$, onde $A_{\mu}$ e $B_{\mu}$ são abertos de $X$ e $T$ respectivamente. Como $K$ é compacto, $K \times t_{0}$ também é compacto, e portanto, está 
contido na união de um número finito dos abertos $A_{\mu} \times B_{\mu}$, sejam

$$
A_{1} \times B_{1}, \ldots, A_{n} \times B_{n}
$$

tais abertos, com $t_{0} \in B_{i}$ para cada $i=1, \ldots, n$. Portanto,

$$
B=B_{1} \cap \ldots \cap B_{n}
$$

é um aberto de $T$ contendo $t_{0}$ e está contido em $g^{-1}(W)$, e como $t_{0}$ é um ponto arbitrário de $g^{-1}(W)$, temos que este é um aberto de $T$, e portanto, $g=\theta(h)$ é contínua.

Consideremos $\theta$ restrita a $Y^{X \times T}$, ou seja, consideremos $\theta$ como sendo a função que associa a uma função contínua $h$ de $X \times T$ em $Y$, uma função contínua $\theta(h)$ de $T$ em $Y^{X}$, novamente, $\theta(h)$ é dada por

$$
\theta(h)(t)=h_{t}, \quad \text { onde } \quad h_{t}(x)=h(x, t) .
$$

Consideremos ainda, a função

$$
\omega: Y^{X} \times X \rightarrow Y
$$

definida por $\omega(f, x)=f(x)$ para cada $f \in Y^{X}$ e $x \in X$.

Proposição 4. A função $\theta: Y^{X \times T} \rightarrow\left(Y^{X}\right)^{T}$ é sobrejetora para todo espaço $T$ se, e somente se, $\omega$ é contínua.

Demonstração. $(\Rightarrow)$ Tomemos em particular $T=Y^{X}$ e $g \in\left(Y^{X}\right)^{T}$ como sendo a aplicação identidade em $Y^{X}$. Então existe $h \in Y^{X \times T}$ tal que $\theta(h)=g$. Como

$$
h(x, f)=[g(f)](x)=f(x)=\omega(f, x)
$$

para todo $x \in X$ e $f \in Y^{X}$, temos que $\omega$ é contínua assim como $h$.

$(\Leftarrow)$ Sejam $g \in\left(Y^{X}\right)^{T}$ e $\chi: X \times T \rightarrow Y^{X} \times X$ dada por

$$
\chi(x, t)=(g(t), x) .
$$

Seja $h=\omega \circ \chi$. Como

$$
[[\theta(h)](t)](x)=h(x, t)=\omega(\chi(x, t))=\omega(g(t), x)=[g(t)](x)
$$


para todo $t \in T$ e $x \in X$, temos que $\theta(h)=g$, e portanto, $\theta$ é sobrejetora.

Proposição 5. Se X é um espaço regular e localmente compacto, então a função $\omega$ é contínua.

Demonstração. Sejam $f \in Y^{X}, x \in X$ e um aberto $V$ de $Y$ contendo $f(x)$. Como $f$ é contínua, $f^{-1}(V)$ é um aberto de $X$ contendo $x$. Como $X$ é um espaço regular e localmente compacto, existe uma vizinhança aberta $U$ de $x$ cujo fecho é compacto e está contido em $f^{-1}(V), x \in U \subset \bar{U} \subset f^{-1}(V)$. Então $W=W(\bar{U}, V)$ é um aberto de $Y^{X}$ contendo $f$. Portanto, $W \times U$ é um aberto de $Y^{X} \times X$ e $\omega(W \times U) \subset V$, como $V$ é um aberto arbitrário de $Y$ temos que $\omega$ é contínua.

A topologia atribuída ao espaço $Y^{X}$ é admissível se a função $\omega$ é contínua, portanto, se $X$ é um espaço regular e localmente compacto, temos que a topologia compacto-aberta é admissível. Pode se mostrar ainda que a topologia compactoaberta é menos fina do que qualquer topologia admissível de $Y^{X}$ para quaisquer $X$ e $Y$. Como corolário do resultado anterior, quando $X$ é um espaço regular e localmente compacto, a topologia compacto-aberta possui a propriedade que descrevemos no início.

Corolário 1. Se $X$ é um espaço regular e localmente compacto, então $\theta: Y^{X \times T} \rightarrow$ $\left(Y^{X}\right)^{T}$ é uma correspodência biunívoca entre os espaços de funções $Y^{X \times T}$ e $\left(Y^{X}\right)^{T}$.

Sob certas condições, temos relações ainda mais fortes entre esses dois espaços.

Lema 1. Se $X$ é um espaço de Hausdorff e $\{U\}$ é uma subbase de $Y$, então $\{W(K, U)\}$ é uma subbase de $Y^{X}$, onde $K$ é compacto de $X$ e $U \in\{U\}$.

Demonstração. Basta mostrarmos que se $K$ é um compacto de $X, V$ é um aberto de $Y$ e $f \in W(K, V)$, então existem compactos $K_{1}, \ldots, K_{m}$ de $X$ e elementos $U_{1}, \ldots, U_{m}$ de $\{U\}$ tais que

$$
f \in W\left(K_{1}, U_{1}\right) \cap \ldots \cap W\left(K_{m}, U_{m}\right) \subset W(K, V) .
$$

Seja $x \in K$. Como $f(x) \in V$ existe um número finito de abertos de $\{U\}$, que denotaremos por $U_{x, 1}, \ldots, U_{x, n_{x}}$, tais que

$$
f(x) \in U_{x, 1} \cap \ldots \cap U_{x, n_{x}} \subset V .
$$

Como $f$ é contínua, existe uma vizinhança $G_{x}$ de $x$ em $X$ tal que

$$
f\left(G_{x}\right) \subset U_{x, 1} \cap \ldots \cap U_{x, n_{x}}
$$


Como $K$ é um espaço Hausdorff compacto, temos que $K$ é regular. Então, existe uma vizinhança aberta $H_{x}$ de $x$ em $K$ tal que seu fecho, $K_{x}=\bar{H}_{x}$, está contido em $G_{x}$. A coleção $\left\{H_{x} \mid x \in K\right\}$ é uma cobertura por abertos do espaço compacto $K$, portanto, existe um número finito de pontos em $K$, que denotaremos por $x_{1}, \ldots, x_{q}$, tais que

$$
K=H_{x_{1}} \cup \ldots \cup H_{x_{q}} .
$$

Temos então que $K_{x_{1}} \cup \ldots \cup K_{x_{q}}$ são compactos e

$$
f\left(K_{x_{j}}\right) \subset f\left(G_{x_{j}}\right) \subset U_{x_{j}, 1} \cap \ldots \cap U_{x_{j}, n_{x_{j}}} \subset V, \quad j=1, \ldots, q,
$$

e portanto,

$$
f \in \bigcap_{j=1}^{q}\left[\bigcap_{i=1}^{n_{j}} W\left(K_{x_{j}}, U_{x_{j}, i}\right)\right] .
$$

Suponhamos que $g \in Y^{X}$ está em $\cap_{j=1}^{q}\left[\cap_{i=1}^{n_{j}} W\left(K_{x_{j}}, U_{x_{j}, i}\right)\right]$. Se $x \in K$, então $x \in H_{j}$ para algum $j$, logo, $x \in K_{j}$. Portanto,

$$
g(x) \in U_{x_{j}, 1} \cap \ldots \cap U_{x_{j}, n_{x_{j}}} \subset V .
$$

Então $g \in W(K, V)$, e

$$
f \in \bigcap_{j=1}^{q}\left[\bigcap_{i=1}^{n_{j}} W\left(K_{x_{j}}, U_{x_{j}, i}\right)\right] \subset W(K, V)
$$

Proposição 6. Se T é um espaço de Hausdorff, então $\theta: Y^{X \times T} \rightarrow\left(Y^{X}\right)^{T}$ é continua.

Demonstração. Como $T$ é um espaço de Hausdorff e $\{W(K, U)\}$ é uma subbase de $Y^{X}$ temos, pelo lema anterior, que a coleção formada pelos conjuntos

$$
W(L, W(K, U))=\left\{g \in\left(Y^{X}\right)^{T} \mid g(L) \subset W(K, U)\right\}
$$

é uma subbase de $\left(Y^{X}\right)^{T}$, onde $L$ é compacto de $T, K$ é compacto de $X$, e $W$ é abertos de $Y$. Pela definição de $\theta$, temos que

$$
\theta^{-1}(W(L, W(K, U)))=\left\{h \in Y^{X \times T} \mid[\theta(h)](L) \subset W(K, U)\right\}
$$


e como

$$
[\theta(h)](L) \subset W(K, U) \Rightarrow[[\theta(h)](L)](K) \subset U \Rightarrow h(K \times L) \subset U,
$$

temos que

$$
\theta^{-1}(W(L, W(K, U)))=\left\{h \in Y^{X \times T} \mid h(K \times L) \subset U\right\}=W(K \times L, U) .
$$

Como $K \times L$ é compacto, temos que $W(K \times L, U)$ é um aberto de $Y^{X \times T}$. E como $\{W(L, W(K, U))\}$ é uma subbase de $\left(Y^{X}\right)^{T}$, segue que $\theta$ é contínua.

Proposição 7. Se $X$ e T são espaços de Hausdorff, então $\theta: Y^{X \times T} \rightarrow\left(Y^{X}\right)^{T}$ é um homeomorfismo de $Y^{X \times T}$ sobre um subespaço de $\left(Y^{X}\right)^{T}$.

Demonstração. Como $\theta$ é injetora e contínua pelo resultado anterior, resta apenas mostrar que $\theta^{-1}$ é contínua em $\theta\left(Y^{X \times T}\right) \subset\left(Y^{X}\right)^{T}$. Para isso, basta mostrarmos que se $J$ é um subespaço compacto de $X \times T$ e $V$ é um aberto de $Y$, então a imagem $\theta[W(J, V)]$ é um aberto de $\theta\left(Y^{X \times T}\right)$. Dado $\psi \in \theta[W(J, V)]$, seja $\phi \in W(J, V)$ tal que $\theta(\phi)=\psi$. Sejam $J_{X}$ e $J_{T}$ as projeções de $J$ em $X$ e $T$, respectivamente. Para cada $z=(x, t) \in J, \operatorname{sejam} U_{z}$ uma vizinhança aberta de $x$ de $J_{X}$ e $U_{z}^{\prime}$ uma vizinhança aberta de $t$ em $J_{T}$, tais que

$$
\phi\left(U_{z} \times U_{z}^{\prime}\right) \subset V
$$

Como $X$ e $T$ são Hausdorff, temos que $X \times T$ é Hausdorff, logo, $J$ é um espaço Hausdorff compacto, assim como $J_{X}$ e $J_{T}$, e portanto, $J_{X}$ e $J_{T}$ são espaços regulares. Portanto, podemos tomar $U_{z}$ e $U_{z}^{\prime}$ de modo que

$$
\phi\left(K_{z} \times L_{z}\right) \subset V
$$

onde $K_{z}$ denota o fecho de $U_{z}$ em $J_{X}$ e $L_{z}$ o fecho de $U_{z}^{\prime}$ em $J_{T}$. A coleção $\left\{\left(U_{z} \times\right.\right.$ $\left.\left.U_{z}^{\prime}\right) \cap J \mid z \in J\right\}$ é uma cobertura por abertos do espaço compacto J. Portanto, existe um número finito de pontos em $J$, que denotaremos por $z_{1}, \ldots, z_{n}$, tais que

$$
J \subset\left(U_{z_{1}} \times U_{z_{1}}^{\prime}\right) \cup \ldots \cup\left(U_{z_{n}} \times U_{z_{n}}^{\prime}\right)
$$

Temos então que $K_{z_{i}}$ e $L_{z_{i}}, i=1, \ldots, n$ são compactos e

$$
\left[\psi\left(L_{z_{i}}\right)\right]\left(K_{z_{i}}\right)=\phi\left(K_{z_{i}} \times L_{z_{i}}\right) \subset V, \quad i=1, \ldots, n .
$$


Portanto,

$$
\psi \in \theta\left(Y^{X \times T}\right) \cap\left\{\bigcap_{i=1}^{n} W\left[L_{z_{i}}, W\left(K_{z_{i}}, V\right)\right]\right\} .
$$

Suponhamos que $\chi \in\left(Y^{X}\right)^{T}$ está em $\theta\left(Y^{X \times T}\right) \cap\left\{\cap_{i=1}^{n} W\left[L_{z_{i}}, W\left(K_{z_{i}}, V\right)\right]\right\}$. Como $\chi \in \theta\left(Y^{X \times T}\right)$, existe $\xi \in Y^{X \times T}$ tal que $\chi=\theta(\xi)$. Se $z=(x, t) \in J$, então $z \in U_{z_{i}} \times U_{z_{i}}^{\prime}$ para $\operatorname{algum} i, \log \mathrm{O}, z \in K_{z_{i}} \times L_{z_{i}}$. E como $x \in K_{z_{i}}, t \in L_{z_{i}}$ e $\chi \in W\left[L_{z_{i}}, W\left(K_{z_{i}}, V\right)\right]$, temos que

$$
\xi(z)=\xi(x, t)=[\chi(t)](x) \in V .
$$

Então $\xi(J) \subset V$. Portanto, $\xi \in W(J, V)$ e $\chi=\theta(\xi) \in \theta[W(J, V)]$. Obtemos então

$$
\psi \in \theta\left(Y^{X \times T}\right) \cap\left\{\bigcap_{i=1}^{n} W\left[L_{z_{i}}, W\left(K_{z_{i}}, V\right)\right]\right\} \subset \theta[W(J, V)] .
$$

E portanto, $\theta[W(J, V)]$ é um aberto de $\theta\left(Y^{X \times T}\right)$.

Como consequência imediata dos resultados anteriores, temos o seguinte resultado.

Teorema 1. Se T é um espaço de Hausdorff e X é um espaço regular e localmente compacto, então $\theta: Y^{X \times T} \rightarrow\left(Y^{X}\right)^{T}$ é um homeomorfismo. 


\section{Capítulo 2}

\section{Variedades Topológicas e o Teorema da Dominação}

Utilizaremos o termo espaço para nos referir a um espaço métrico separável, e aplicação para uma função contínua entre dois espaços métricos separáveis. Denotaremos por $X^{I}$ o conjunto dos caminhos em um espaço $X$.

\subsection{Simplexos e complexos simpliciais}

O subconjunto $\sigma$ do cubo de Hilbert formado pelos pontos da forma

$$
x=t_{0} x_{0}+\ldots+t_{n} x_{n}, \quad \text { onde } t_{i} \geq 0, \quad t_{0}+\ldots+t_{n}=1 \quad \text { e } \quad x_{0}, \ldots, x_{n} \in I^{\omega}
$$

é o n-simplexo gerado por $\left\{x_{0}, \ldots, x_{n}\right\}$. Os pontos $x_{0}, \ldots, x_{n}$ são os vértices de $\sigma$, os reais $t_{0}, \ldots, t_{n}$ são as coordenadas baricêntricas de $x$ em relação a $x_{0}, \ldots, x_{n}$, o inteiro $n$ é a dimensão de $\sigma$. Um simplexo gerado por um subconjunto de $\left\{x_{0}, \ldots, x_{n}\right\}$ é uma face de $\sigma$, a união das faces próprias de $\sigma$ é o seu bordo $(B d \sigma)$, e $\sigma-B d \sigma$ é o interior de $\sigma$.

Nas definições abaixo, utilizaremos a nomenclatura encontrada em [19].

Um complexo simplicial $\mathcal{K}$ é uma coleção de simplexos tais que:

1. toda face de um simplexo de $\mathcal{K}$ é um simplexo de $\mathcal{K}$,

2. a interseção de dois simplexos de $\mathcal{K}$ é uma face de ambos.

Um subcomplexo de $\mathcal{K}$ é uma subcoleção de $\mathcal{K}$ que contém todas as faces de seus elementos. Um subcomplexo de $\mathcal{K}$ que contém todos os simplexos de $\mathcal{K}$ de dimensão menor ou igual a $p$ é o p-esqueleto de $K, K^{p}$, consequentemente, $K^{0}$ é o conjunto 
formado pelos vértices dos elementos de $\mathcal{K}$. Um complexo simplicial $\mathcal{K}$ é localmente finito se cada vértice de $\mathcal{K}$ pertence a um número finito de simplexos de $\mathcal{K}$.

O conjunto $P$ dado por uma união enumerável de elementos de um complexo simplicial $\mathcal{K}$ munido com uma topologia de modo que $A \subset P$ é aberto se, e somente se, $A \cap \sigma$ é aberto em $\sigma$ para todo $\sigma \in \mathcal{K}$ é um poliedro simplicial. Um conjunto $P_{0}$ tal que, $P_{0}$ é dado por uma união enumerável de elementos de um subcomplexo de $\mathcal{K}$, tais elementos estão contidos em $P$, e $P_{0}$ é munido com a topologia induzida por $P$, é um subpoliedro simplicial de $P$. Um espaço homeomorfo a um poliedro simplicial é um poliedro.

Um complexo simplicial $\mathcal{K}^{\prime}$ é uma subdivisão do complexo simplicial $\mathcal{K}$ se todo simplexo de $\mathcal{K}$ é uma união de simplexos de $\mathcal{K}^{\prime}$. Em particular, se substituirmos cada simplexo de $\mathcal{K}$ por sua subdivisão baricêntrica, o complexo simplicial $\mathcal{K}^{\prime}$ obtido é a subdivisão baricêntrica de $\mathcal{K}$.

Um complexo simplicial abstrato é uma coleção $\mathcal{S}$ de conjuntos não vazios tal que todo subconjunto não vazio de um elemento de $\mathcal{S}$ pertence a $\mathcal{S}$. Um elemento $A$ de $\mathcal{S}$ é um simplexo de $\mathcal{S}$, sua dimensão é um menos o seu número de elementos. Cada subconjunto de $A$ é uma face de $A$. A dimensão de $\mathcal{S}$ é a maior das dimensões de seus elementos, ou infinito se esse número não existe. O conjunto dos vértices de $\mathcal{S}$ é a união dos elementos de $\mathcal{S}$ com apenas um ponto. Uma subcoleção de $\mathcal{S}$ que é também é um complexo simplicial abstrato é um subcomplexo de $\mathcal{S}$. Os complexos abstratos $\mathcal{S}$ e $\mathcal{T}$ são isomorfos se existe uma bijeção $f$ do conjunto dos vértices de $\mathcal{S}$ no conjunto dos vértices de $\mathcal{T}$ tal que $\left\{a_{0}, \ldots, a_{n}\right\} \in \mathcal{S} \Leftrightarrow\left\{f\left(a_{0}\right), \ldots, f\left(a_{n}\right)\right\} \in \mathcal{T}$. Se $\mathcal{K}$ é um complexo simplicial, $V$ é o conjunto dos vértices de $\mathcal{K}$ e $K$ é a coleção de todos subconjuntos $\left\{a_{0}, \ldots, a_{n}\right\}$ de $V$ tais que $a_{0}, \ldots, a_{n}$ geram um simplexo de $\mathcal{K}$, então $K$ é o esquema de $\mathcal{K}$. Se o complexo abstrato $\mathcal{S}$ é isomorfo ao esquema do complexo simplicial $\mathcal{K}$, temos que $\mathcal{K}$ é uma realização geométrica de $\mathcal{S}$.

\subsection{Coberturas estrela finitas e nervo de uma co- bertura}

Uma cobertura $\mathcal{U}=\left\{U_{\mu}\right\}_{\mu \in M}$ de $X$ é estrela finita se, para todo $\mu \in M$, temos que $U_{\mu} \cap U_{\lambda} \neq \emptyset$ apenas para um número finito de $\lambda$ 's. Lembrando que, uma cobertura $\mathcal{U}=\left\{U_{\mu}\right\}_{\mu \in M}$ de $X$ é localmente finita se, para todo $x \in X$, existe uma vizinhança $W$ de $x$, tal que $W \cap U_{\mu} \neq \emptyset$ apenas para um número finito de $\mu$ 's, segue portanto, que toda cobertura por abertos estrela finita de $X$ é localmente finita.

Um espaço possui a propriedade estrela finita se toda cobertura por abertos deste 
espaço possui um refinamento aberto estrela finito. Lembrando que, um espaço é paracompacto se toda cobertura por abertos deste espaço possui um refinamento aberto localmente finito, segue portanto, que todo espaço que possui a propriedade estrela finito é paracompacto.

O resultado a seguir é uma adaptação de [17].

Proposição 8. Seja $\mathcal{U}=\left\{U_{\lambda}\right\}$ uma cobertura por abertos de um espaço $X$, então existe $\mathcal{V}=\left\{V_{n}\right\}_{n \in \mathbb{N}}$ uma cobertura por abertos de $X$ que refina $\mathcal{U}$, enumerável $e$ estrela finita.

Demonstração. Pela proposição 1, um espaço métrico separável é homeomorfo a um subespaço do cubo de Hilbert, que é um espaço métrico compacto. Portanto, basta provarmos o teorema para um subespaço arbitrário de um espaço métrico compacto. Sejam $R$ um espaço métrico compacto, $A$ um subespaço de $R$ e $\mathcal{U}=\{U\}$ uma cobertura de $A$ formado por subconjuntos abertos de $A$. Para cada $U$, seja $U^{\prime}$ um subconjunto aberto de $R$ tal que $U^{\prime} \cap A=U$. Então $G=\cup U^{\prime}$ é um subconjunto aberto de $R$ contendo $A$, e $\mathcal{U}^{\prime}=\left\{U^{\prime}\right\}$ é uma cobertura por abertos de $G$. Se $G$ é fechado em $R$, então $G$ é compacto, e portanto, existe um número finito de elementos de $\mathcal{U}^{\prime}=\left\{U^{\prime}\right\}$ que formam uma cobertura por abertos de $G$, fazendo a intersecção desses elementos com $A$, obtemos uma cobertura por abertos de $A$ que é finita, logo, enumerável e estrela finita, e esta cobertura refina $\mathcal{U}$. Caso contrário, consideremos a cobertura $\mathcal{U}^{\prime}=\left\{U^{\prime}\right\}$, como $G$ é um espaço métrico, temos que toda cobertura por abertos de $G$ admite uma subcobertura enumerável, tomemos então uma subcobertura $\mathcal{G}^{\prime}=\left\{G_{i}^{\prime}\right\}_{i \geq 1}$ de $\mathcal{U}^{\prime}$. Sejam $G_{i}^{\prime \prime}=\cup_{j=1}^{i} G_{j}^{\prime}$ e a cobertura de $G$ dada por $\mathcal{G}^{\prime \prime}=\left\{G_{i}^{\prime \prime}\right\}_{i \geq 1}$. Tomemos $G_{0}^{\prime \prime}=\{b\}$, onde $b$ é um elemento de $G_{1}^{\prime \prime}$. O conjunto $R \backslash G_{i}^{\prime \prime}$ é fechado, logo compacto, portanto, para todo $i \geq 1$, podemos definir

$$
d_{i}^{\prime \prime}=d\left(b, R \backslash G_{i}^{\prime \prime}\right), \quad \text { a distância de } \quad b \quad \text { a } \quad R \backslash G_{i}^{\prime \prime} .
$$

Sejam ainda, $G_{0}=\{b\}, d_{0}=0$ e para $i \geq 1, d_{i}=d_{i-1}^{\prime \prime}+\left(d_{i}^{\prime \prime}-d_{i-1}^{\prime \prime}\right) / 2$ e

$$
G_{i}=\left\{x \in R \mid x \in G_{i}^{\prime \prime} \text { e } d\left(x, R \backslash G_{i}^{\prime \prime}\right)>d_{i} / 2\right\}
$$

Como $G_{i}^{\prime \prime} \subset G_{i+1}^{\prime \prime}$ e $d_{i}<d_{i+1}$, segue que $G_{i} \subset G_{i+1}$, e pela construção de $G_{i}$, segue ainda que $\bar{G}_{i} \subset G_{i+1}$ para todo $i$. Temos então que $G$ é dado pela união de uma sequência de abertos, $G=\cup_{i=0}^{\infty} G_{i}$, tais que $\bar{G}_{i} \subset G_{i+1}$. Para cada $i>0, \bar{G}_{i}$ é compacto e está contido em $G$, e portanto, é coberto por um número finito de $U^{\prime}$ 's, 
os denotaremos por $U_{i 1}^{\prime}, \ldots, U_{i h(i)}^{\prime}$. Seja

$$
V_{i j}^{\prime}=U_{i j}^{\prime} \cap\left(G_{i+1}-\bar{G}_{i-1}\right)
$$

Então $\left\{V_{i j}^{\prime}\right\}, i=1,2, \ldots$ e $j=1,2, \ldots, h(i)$, é um refinamento de $\mathcal{U}^{\prime}$, aberto, enumerável e estrela finito, denotemos por $\mathcal{V}^{\prime}=\left\{V_{m}^{\prime}\right\}_{m \in \mathbb{N}}$ uma enumeração desta cobertura. Seja $V_{m}=V_{m}^{\prime} \cap A$, então $\mathcal{V}=\left\{V_{m}\right\}_{m \in \mathbb{N}}$ é uma cobertura por abertos de $A$ que refina $\mathcal{U}$, enumerável e estrela finita.

Dados um espaço $X$ e uma cobertura por abertos $\mathcal{U}=\left\{U_{\mu}\right\}_{\mu \in M}$ de $X$ tal que $\mu_{i} \neq$ $\mu_{j} \Rightarrow U_{\mu_{i}} \neq U_{\mu_{j}}$, consideremos a coleção formada pelos conjuntos $\left\{U_{\mu_{0}}, \ldots, U_{\mu_{n}}\right\}$ onde $U_{\mu_{i}}$ são tais que $\cap_{i=0}^{n} U_{\mu_{i}} \neq \emptyset$, então esta coleção é um complexo simplicial abstrato, onde um conjunto da forma $\left\{U_{\mu_{0}}, \ldots, U_{\mu_{n}}\right\}$ é um n-simplexo.

Definição 1. O poliedro simplicial dado pela união dos elementos de uma realização geométrica do complexo simplicial abstrato descrito acima é o nervo da cobertura $\mathcal{U}$.

Dados um espaço $X$ e uma cobertura por abertos $\mathcal{U}=\left\{U_{\mu}\right\}_{\mu \in M}$ de $X$, enumerável e estrela finita. Seja $P$ o nervo da cobertura $\mathcal{U}$, como a cobertura é enumerável, temos que $P$ é um poliedro simplicial com uma quantidade enumerável de vértices. Sejam $\left\{p_{i}\right\}_{i \in \mathbb{N}}$ uma enumeração destes vértices. A aplicação baricêntrica é a aplicação $g: X \rightarrow P$ dada por

$$
g(x)=\sum_{i} t_{i}(x) \cdot p_{i}, \quad \text { onde } \quad t_{i}(x)=\frac{d\left(x, X-U_{i}\right)}{\sum_{i} d\left(x, X-U_{i}\right)} .
$$

Notemos que, como $\mathcal{U}$ é uma cobertura de $X$, existe pelo menos um $U_{j}$ tal que $x \in U_{j}$, e como os $U_{i}$ 's são abertos, $X-U_{j}$ é fechado e $d\left(x, X-U_{j}\right)>0$, e portanto, $\sum_{i} d\left(x, X-U_{i}\right) \geq d\left(x, X-U_{j}\right)>0$. Além disso, temos que para todo $x \in X$, $\sum_{i} t_{i}(x)=1$.

\subsection{Dimensão de um espaço métrico}

Seja $n \geq-1$ um inteiro. A ordem de uma família $\mathcal{A}$ de conjuntos é menor ou igual a $n$ se, para quaisquer $n+2$ conjuntos de $\mathcal{A}$, sua interseção é vazia. Se $n \geq 0$, dizemos que $\mathcal{A}$ possui ordem $n$ se $\mathcal{A}$ possui ordem $\leq n$ mas não possui ordem $\leq n-1$. Temos, por exemplo, se uma família de conjuntos possui ordem -1 , então ela é vazia 
ou igual a $\{\emptyset\}$. Uma família de conjuntos não vazios possui ordem zero se, e somente se, seus elementos são dois a dois disjuntos.

Sejam $X$ um espaço métrico e $n \geq-1$ um inteiro, dizemos que a dimensão de $X$ é menor ou igual a $n(\operatorname{dim} X \leq n)$ se, e somente se, toda cobertura por abertos finita de $X$ possui um refinamento de ordem menor ou igual a $n$, e $\operatorname{dim} X=n$ se $\operatorname{dim} X \leq n$ e não vale que $\operatorname{dim} X \leq n-1$. Se não vale que $\operatorname{dim} X \leq n$ para nenhum inteiro $n$, então $X$ possui dimensão infinita $(\operatorname{dim} X=\infty)$.

Se $\mathcal{A}$ é uma cobertura de um espaço métrico compacto, definimos a norma de $\mathcal{A}$ como o $\sup _{A \in \mathcal{A}}\{\operatorname{diam} A\}$

\subsection{Retratos e extensões}

Se $f: X \rightarrow Y$ é uma aplicação e $A$ é um subespaço de $X$, então $f$ define uma única aplicação $g: A \rightarrow Y$ tal que $g(x)=f(x)$ para todo $x \in A, g$ é a restrição de $f$ em $A\left(g=f_{\backslash A}\right)$ e $f$ é a extensão de $g$ sobre $X$.

Se $i: A \rightarrow X$ é a inclusão, definida por $i(a)=a$ para todo $a \in A$, então $g=f_{\downarrow A}$ é equivalente a relação comutativa $f \circ i=g$ no diagrama:

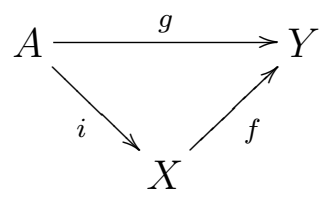

Consideremos o caso especial em que $Y=A$ e $g=i d$, a aplicação identidade em $A$. Então $f_{\downarrow A}=i d$ é equivalente a $f \circ i=i d$ no diagrama:

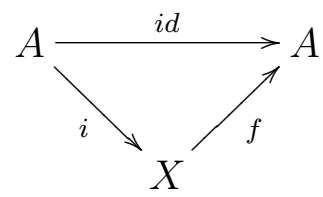

neste caso, $A$ é um retrato de $X$ e $f$ é uma retração de $X$ sobre $A$.

Exemplo 1. Seja $B^{n}=\left\{x \in E^{n} \mid\|x\| \leq 1\right\}$ a n-bola em $\mathbb{R}^{n}$, o produto cartesiano de $n$ cópias de $\mathbb{R}$, então $B^{n}$ é um retrato de $\mathbb{R}^{n}$.

Exemplo 2. Seja $S^{n-1}=\left\{x \in E^{n} \mid\|x\|=1\right\}$ a esfera em $\mathbb{R}^{n}$, então $S^{n-1}$ não é um retrato de $\mathbb{R}^{n}$.

O subespaço $A$ é um retrato de vizinhança (NR) de $X$ se $A$ é retrato de um aberto de $X$ que contém $A$. 
Um subespaço $A$ de um espaço $X$ possui a propriedade da extensão (PE) em $X$ com respeito ao espaço $Y$ se toda aplicação $f: A \rightarrow Y$ possui uma extensão em $X$. O subespaço $A$ possui a propriedade da extensão absoluta (PEA) em $X$, se possui a $\mathrm{PE}$ em $X$ com respeito a todo espaço $Y$.

Proposição 9. O subespaço $A$ é um retrato de $X$ se, e somente se, $A$ possui a propriedade da extensão absoluta em $X$.

\subsection{Homotopia e extensão de homotopias}

Uma homotopia é uma aplicação $H: X \times I \rightarrow Y$, onde $I$ é o intervalo unitário, usaremos também a notação $h_{t}: X \rightarrow Y$, onde para cada $t \in I, h_{t}$ é a aplicação dada por $h_{t}(x)=H(x, t)$, e assumiremos portanto, que temos continuidade em ambas as variáveis $x$ e $t$. Uma homotopia de uma aplicação $f$ é uma homotopia $h_{t}$ tal que $h_{0}=f$. As aplicações $f$ e $g$ são homotópicas se existe uma homotopia $h_{t}$ tal que $h_{0}=f$ e $h_{1}=g$.

Se a aplicação identidade $i d: X \rightarrow X$ é homotópica a $i \circ r: X \rightarrow X$, onde $r: X \rightarrow A$ é uma retração e $i: A \rightarrow X$ é a inclusão, temos que $A$ é um retrato de deformação de $X$.

Exemplo 3. A esfera $S^{n-1}$ em $\mathbb{R}^{n}$ é um retrato de deformação de $\mathbb{R}^{n}-\{0\}$. E se removermos de $\mathbb{R}^{n}$ um ponto a tal que $\|a\| \neq 0$ e $\|a\| \neq 1$, então $S^{n-1}$ é um retrato de $\mathbb{R}^{n}-(\{0\} \cup\{a\})$ mas não é um retrato de deformação deste conjunto.

Se existem aplicações $f: X \rightarrow Y$ e $g: Y \rightarrow X$ tais que $f \circ g$ é homotópica a identidade em $Y$, temos que o espaço $X$ domina $Y$. Se além disso $g \circ f$ é homotópica a identidade em $\mathrm{X}$, temos que $X$ e $Y$ são homotopicamente equivalentes.

Se $H$ é uma homotopia da inclusão $i: A \rightarrow Y$, então $H$ é uma deformação de $A$ em $Y$. Se tivermos ainda que $h_{1}$ é uma aplicação tomando valores em $B \subset Y$, então $H$ é uma contração de $A$ em $B$, e se $B$ consiste em um único ponto, então $A$ é contrátil em $Y$.

Exemplo 4. Todo subconjunto convexo $A$ de um espaço vetorial $V$ é contrátil em si mesmo.

Um subespaço $A$ de um espaço $X$ possui a propriedade da extensão de homotopia (PEH) em $X$ com respeito ao espaço $Y$ se toda homotopia $H: A \times$ $I \rightarrow Y$ de $f_{\downarrow A}$, onde $f: X \rightarrow Y$ é uma aplicação qualquer, possui uma extensão 
$G: X \times I \rightarrow Y$ tal que $G(x, 0)=f(x)$.

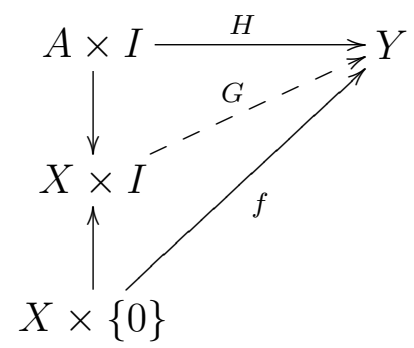

O subespaço $A$ possui a propriedade da extensão de homotopia absoluta (PEHA) em $X$ se possui a PEH em $X$ com respeito a todo espaço $Y$.

\subsection{Teorema da Dominação}

Denotaremos por $(Y, B)$ o par formado por um espaço $Y$ e um subconjunto fechado $B$ de $Y$.

Definição 2. Um espaço $X$ é um retrato absoluto (AR) se para todo homeomorfismo $h$ de $X$ sobre $h(X)$, com $h(X)$ um subconjunto fechado de $Y$, temos que $h(X)$ é um retrato de $Y$. O espaço $X$ é um retrato de vizinhança absoluto (ANR) se para todo homeomorfismo $h$ de $X$ sobre $h(X)$, com $h(X)$ um subconjunto fechado de $Y$, temos que $h(X)$ é um retrato de vizinhança de $Y$.

Proposição 10. Se $Y$ é um ANR, então todo fechado $A$ de um espaço $X$ possui a PEH em $X$ com respeito a $Y$.

A demonstração do resultado anterior pode ser encontrada em [8] página 86.

Proposição 11. Sejam $X$ um ANR e A um subespaço fechado de $X$, então são equivalentes:

1. A possui a PEHA em $X$

2. $X \times\{0\} \cup A \times I$ é um retrato de $X \times I$

3. $X \times\{0\} \cup A \times I$ é um $A N R$

4. A é um $A N R$

A demonstração do resultado anterior pode ser encontrada em [13].

Proposição 12. Um espaço compacto $X$ é um compacto ANR se, e somente se, existe um mergulho $i: X \rightarrow I^{\omega}$ tal que $i(X)$ é um retrato de vizinhança de $I^{\omega}$. 
Demonstração. $(\Rightarrow)$ Pela proposição 1, podemos mergulhar todo compacto ANR em $I^{\omega}$, e como $I^{\omega}$ é um espaço métrico separável temos que o compacto ANR é mergulhado como um retrato de vizinhança.

$(\Leftarrow)$ Suponhamos que $X$ é um espaço métrico compacto e existe um mergulho $i$ de $X$ em $I^{\omega}$ tal que $i(X)$ é um retrato de vizinhança de $I^{\omega}$. Seja $p_{j}: I^{\omega} \rightarrow[-1 / j, 1 / j]$ a aplicação que associa a uma sequência em $I^{\omega}$ sua j-ésima coordenada. Seja $Y$ um espaço métrico separável, $A$ um subconjunto de $Y$ e $h$ um homeomorfismo de $A$ sobre $X$. Pelo Teorema da Extensão de Tietze a aplicação $p_{j} \circ i \circ h: A \rightarrow[-1 / j, 1 / j]$ pode ser estendida a uma aplicação $h_{j}: Y \rightarrow[-1 / j, 1 / j]$. Seja $H: Y \rightarrow I^{\omega}$ dada por $H(y)=\left(h_{1}(y), h_{2}(y), \ldots\right)$, então a restrição de $H$ a $A$ é a aplicação $i \circ h$. Como $i \circ h(A)=i(X)$, existe um aberto $U$ em $I^{\omega}$ contendo $i \circ h(A)$ e uma retração $r: U \rightarrow i \circ h(A)$. Consideremos o aberto $H^{-1}(U) \subseteq Y$ que contém $A$, e a retração $h^{-1} \circ i^{-1} \circ r \circ H: H^{-1}(U) \rightarrow A$, então temos que $A$ é um retrato de vizinhança de $Y$, e portanto, $X$ é um compacto ANR.

Lema 2. Se $X$ é um espaço n-dimensional dominado por um poliedro simplicial $P$, dado pela união dos elementos de um complexo simplicial $\mathcal{K}$, então $X$ é dominado por $\left|\mathcal{K}^{n}\right|$, o poliedro dado pela união dos elementos de $\mathcal{K}^{n}$

A demonstração do resultado anterior pode ser encontrada em [1]. O resultado a seguir é uma adaptação de [4] e [12].

Teorema 2 (Teorema da Dominação). Seja B um compacto ANR n-dimensional, então $B$ é dominado por um poliedro $P$ n-dimensional por meio das aplicações $f$ : $P \rightarrow B$ e $g: B \rightarrow P$, onde $g$ é a aplicação baricêntrica e $P$ é o nervo de uma cobertura por abertos de $B$.

Demonstração. Como $B$ é um compacto ANR, existe um mergulho $i: B \rightarrow I^{\omega}$ tal que $i(B)$ é um retrato de um aberto $U$ de $I^{\omega}$ contendo $i(B)$. Sem perda de generalidade, identificaremos $i(B)$ por $B$. Seja $r: U \rightarrow B$, a retração de $U$ em $B$. Seja $\mathcal{U}=\left\{U_{\lambda}\right\}$ uma cobertura por abertos de $B$, então $\mathcal{U}^{\prime}=\left\{r^{-1}\left(U_{\lambda}\right)\right\}$ é uma cobertura por abertos de $U$. Para cada $u \in U$, seja $\eta=\eta(u)>0$ tal que $S(u, \eta)=$ $\left\{x \in I^{\omega} \mid d(x, u)<\eta\right\} \subset r^{-1}\left(U_{\lambda}\right)$ para algum $U_{\lambda}$. Seja $\mathcal{U}^{\prime \prime}=\{S(u, \eta / 2) \cap B\}$, $\mathcal{U}^{\prime \prime}$ é um refinamento de $\mathcal{U}$. Pela proposição 8, existe uma cobertura por abertos $\mathcal{V}=\left\{V_{n}\right\}_{n \in \mathbb{N}}$, enumerável, tal que $\mathcal{V}$ é um refinamento de $\mathcal{U}^{\prime \prime}$ e para cada $i \in \mathbb{N}$, temos que $V_{i} \cap V_{j} \neq \emptyset$ apenas para um número finito de $j$ 's. Então para todo $V_{n}$ existe $u_{n}$ tal que $V_{n} \subset\left(S\left(u_{n}, \eta\left(u_{n}\right) / 2\right) \cap B\right)$. Sejam $P$ o nervo de $\mathcal{V}$ e $p_{n}$ o vértice correspondente a $V_{n}$. Sejam $g: B \rightarrow P$ a aplicação baricêntrica e $f=r \circ \phi: P \rightarrow B$, onde $\phi: P \rightarrow U$ é obtida associando $p_{n}$ a $u_{n} \in V_{n}$ e estendendo-se linearmente a todo 
$P$. Vamos mostrar que $P$ domina $B$ por meio das aplicações $f$ e $g$. Primeiro, vamos mostrar que $\phi$ está bem definida verificando que $\phi(P) \subset U$. Dado $\sigma=\left(p_{n_{1}}, \ldots, p_{n_{r}}\right)$ um simplexo contido em $P$, então $\sigma \subset\{s \in P \mid s=g(b), b \in B\}$. Sejam $V_{n_{1}}, \ldots, V_{n_{r}}$ os elementos de $\mathcal{V}$ aos quais os vértices $p_{n_{1}}, \ldots, p_{n_{r}}$ correspondem, e $\eta_{n_{i}}=\eta\left(u_{n_{i}}\right)$ os respectivos $\eta$-números. Para cada $b \in g^{-1}(\sigma)$ temos que os elementos de $\mathcal{V}$ que o contém estão entre os abertos $V_{n_{1}}, \ldots, V_{n_{r}}$. Suponhamos sem perda de generalidade que $\eta_{n_{1}} \geq \eta_{n_{i}}$, para todo $i \in\{1, \ldots, r\}$, então

$$
b \in V_{n_{i}} \subset\left(S\left(u_{n_{i}}, \eta_{n_{i}} / 2\right) \cap B\right) \Rightarrow d\left(b, u_{n_{i}}\right)<\left(\eta_{n_{i}}\right) / 2 \leq\left(\eta_{n_{1}}\right) / 2,
$$

e portanto,

$$
d\left(u_{n_{1}}, u_{n_{i}}\right)<\eta_{n_{1}} \quad \text { e } \quad u_{n_{i}} \in S\left(u_{n_{1}}, \eta_{n_{1}}\right) \quad(*) .
$$

O ponto $b$ é aplicado por $g$ no simplexo de $P$ gerado por $p_{n_{1}}, \ldots, p_{n_{r}}$,

$$
g(b)=\sum_{i=1}^{r} t_{i}(b) \cdot p_{n_{i}}, \quad \text { onde } \quad t_{i}(b)=\frac{d\left(b, X-U_{i}\right)}{\sum_{i} d\left(b, X-U_{i}\right)}
$$

e este simplexo é aplicado por $\phi$ sobre o simplexo em $I^{\omega}$ gerado por $u_{n_{1}}, \ldots, u_{n_{r}}$, $\log \mathrm{O}$

$$
\phi(g(b))=\phi\left(\sum_{i=1}^{r} t_{i}(b) \cdot p_{n_{i}}\right)=\sum_{i=1}^{r} t_{i}(b) \cdot \phi\left(p_{n_{i}}\right)=\sum_{i=1}^{r} t_{i}(b) \cdot u_{n_{i}},
$$

e, por $(*)$, temos que

$$
d\left(\phi(g(b)), u_{n_{1}}\right)=d\left(\sum_{i=1}^{r} t_{i}(b) \cdot u_{n_{i}}, u_{n_{1}}\right) \leq \sum_{i=1}^{r} t_{i}(b) \cdot d\left(u_{n_{i}}, u_{n_{1}}\right)<\sum_{i=1}^{r} t_{i}(b) \cdot \eta_{n_{1}}=\eta_{n_{1}}
$$

e portanto,

$$
\phi(g(b))=\phi(s) \in S\left(u_{n_{1}}, \eta_{n_{1}}\right),
$$

$\log \mathrm{O}$

$$
\phi(\sigma) \subset S\left(u_{n_{1}}, \eta_{n_{1}}\right) \subset r^{-1}\left(U_{\lambda}\right), \subset U,
$$

e portanto, temos que $\phi(P) \subset U$. Como $I^{\omega}$ é convexo, para todo $x$, podemos unir $x$ e $\phi(g(x))$ por um segmento. Como $x$ e $\phi(g(x)) \in S\left(u_{n_{1}}, \eta_{n_{1}}\right)$, o segmento que une $x$ e $\phi(g(x))$ assume valores em $U$, e a composição com $r$ nos fornece uma homotopia em $B$ entre $i d_{B}$ e $r \circ \phi \circ g=f \circ g$, portanto, $P$ domina $B$ por meio das aplicações $f$ e $g$. E pelo lema anterior, temos que se $P$ é obtido a partir de um complexo simplicial $\mathcal{K}$, então $B$ é dominado por $\left|\mathcal{K}^{n}\right|$. 
Denotemos por $H^{*}(X ; \mathbb{K})$ os grupos de cohomologia singular de $X$ com coeficientes em $\mathbb{K}$.

Corolário 2. Se X é um compacto $A N R$ e $\mathbb{K}$ é um corpo, então $H^{*}(X ; \mathbb{K})$ é um espaço vetorial graduado tal que cada $H^{p}(X ; \mathbb{K})$ é finitamente gerado e $H^{p}(X ; \mathbb{K})$ é trivial exceto para um número finito de inteiros p's.

Demonstração. Pelo resultado anterior, temos que existem um poliedro $P$ e aplicações $f: P \rightarrow X$ e $g: X \rightarrow P$ tais que $f \circ g$ é homotópica à aplicação identidade $i d_{X}: X \rightarrow X$. Portanto,

$$
g^{*} \circ f^{*}=(f \circ g)^{*}=\left(i d_{X}\right)^{*}
$$

que é a identidade em $H^{*}(X ; \mathbb{K})$. Então $f^{*}: H^{*}(X ; \mathbb{K}) \rightarrow H^{*}(P ; \mathbb{K})$ é um isomorfismo de $H^{*}(X ; \mathbb{K})$ no subespaço $f^{*}\left(H^{*}(X ; \mathbb{K})\right)$ de $H^{*}(P ; \mathbb{K})$. E como $P$ é um poliedro, temos que $H^{p}(P ; \mathbb{K})$ é finitamente gerado e $H^{p}(P ; \mathbb{K})$ é trivial exceto para um número finito de inteiros $p$ 's, e portanto, o mesmo vale para $H^{*}(X ; \mathbb{K})$.

\subsection{Variedades topológicas}

Definição 3. Um espaço métrico separável conexo $B$ é uma n-variedade topológica se, para cada $b \in B$, existe um aberto $U$ de $B$ contendo $b$ e um homeomorfismo $h: E^{n} \rightarrow U$. Nesse caso, $U$ é uma vizinhança euclidiana de $b$, ou ainda, uma n-célula.

Proposição 13. Todo subconjunto localmente contrátil e compacto de $\mathbb{R}^{n}$ é um $A N R$.

A demonstração do resultado anterior pode ser encontrada em [6, p. 118]

Corolário 3. Toda n-variedade topológica compacta é um ANR.

Pelo corolário anterior, podemos aplicar o teorema da dominação a toda nvariedade topológica compacta $B$, permitindo assim, obter um polidero n-dimensional $P$ que domine $B$.

A seguir, estabelecemos algumas propriedades que utilizaremos no próximo capítulo.

Lema 3. Sejam $M$ uma n-variedade e $D \subset M$ uma n-célula fechada. Então, para todo $p \in M$, existe $D_{2} \subset M$, n-célula fechada tal que $D \cup\{p\} \subset$ Int $D_{2}$.

A demonstração do resultado anterior pode ser encontrada em [9]. 
Lema 4. Sejam $M$ uma n-variedade topológica e $x_{1}, \ldots, x_{q}$ pontos em $M$. Então existe uma n-célula topológica fechada $C \subset M$ tal que $x_{1}, \ldots, x_{q}$ pertencem ao interior de $C$.

Demonstração. Seja $C_{1} \subset B$ uma n-célula fechada contendo $x_{1}$ em seu interior, pelo resultado anterior, existe $C_{2} \subset M$ uma n-célula fechada tal que $C_{1} \cup\left\{x_{2}\right\} \subset$ Int $C_{2}$. Repetindo este argumento, obtemos $C_{q} \subset B$ uma n-célula fechada contendo $x_{1}, \ldots, x_{q}$ em seu interior. 


\section{Capítulo 3}

\section{Espaços fibrados}

\subsection{O conceito de espaço fibrado}

Definição 4. Sejam $p: E \rightarrow B$ uma aplicação, $\Omega_{p}=\left\{(e, \omega) \subset E \times B^{I} \mid \omega(0)=\right.$ $p(e)\}$ e $\tilde{p}: E^{I} \rightarrow \Omega_{p}$ uma aplicação que a cada $\tau \in E^{I}$ associa o par $(\tau(0), p \tau)$. Uma função levantamento de p é uma aplicação $\lambda: \Omega_{p} \rightarrow E^{I}$ tal que $\tilde{p} \circ \lambda=i d_{\Omega_{p}}$, ou seja, $[\lambda(e, \omega)](0)=e$ e $p(\lambda(e, \omega))=\omega$.

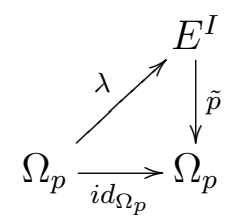

$A$ aplicação $p: E \rightarrow B$ satisfaz a propriedade de levantamento de caminhos - PLC se admite uma função levantamento.

Definição 5. A terna $(E, p, B)$ é um espaço fibrado se $p: E \rightarrow B$ é uma aplicação que admite uma função levantamento, ou seja, se p satisfaz a PLC. Dado b $\in$ B, o conjunto $p^{-1}(b)$ é a fibra de $b$.

A função levantamento $\lambda$ do espaço fibrado $(E, p, B)$ induz uma aplicação

$$
\begin{aligned}
& \tilde{\lambda}: E^{I} \longrightarrow E^{I} \\
& \alpha \longmapsto \lambda(\alpha(0), p(\alpha)) \text {. }
\end{aligned}
$$

Os resultados encontrados neste capítulo são uma adaptação de [10], [14], [16] e $[7]$.

Lema 5. Existe uma homotopia $h_{s}: E^{I} \rightarrow E^{I}$ tal que $h_{0}=\tilde{\lambda}, h_{1}=i d_{E^{I}} e$ $p\left(h_{s}(\alpha(t))\right)=p(\alpha(t))$. 
Demonstração. Para $\alpha \in E^{I}, s \in I$, seja $\alpha_{s} \in E^{I}$ o caminho dado por

$$
\alpha_{s}(t)= \begin{cases}\alpha(t), & 0 \leq t \leq s \\ \alpha(s), & s \leq t \leq 1\end{cases}
$$

Sejam $\omega=p \alpha$ e $\omega_{1-s}$ o caminho definido por

$$
\omega_{1-s}(t)=\left\{\begin{array}{lr}
\omega(s+t), & 0 \leq t \leq 1-s \\
\omega(1), & 1-s \leq t \leq 1
\end{array}\right.
$$

Seja $h_{s}: E^{I} \rightarrow E^{I}$ dada por

$$
\left[h_{s}(\alpha)\right](t)= \begin{cases}\alpha_{s}(t), & 0 \leq t \leq s \\ {\left[\lambda\left(\alpha(s), \omega_{1-s}\right)\right](t-s),} & s \leq t \leq 1\end{cases}
$$

A função $h_{s}$ é dada por uma composição de funções contínuas, e além disso, para $t=s$ segue que

$$
\begin{gathered}
\alpha_{s}(t)=\alpha(s) \mathrm{e} \\
{\left[\lambda\left(\alpha(s), \omega_{1-s}\right)\right](t-s)=\left[\lambda\left(\alpha(s), \omega_{1-s}\right)\right](0)=\alpha(s),}
\end{gathered}
$$

logo, $h_{s}$ é contínua, e portanto, uma homotopia. E temos também que

$$
\begin{gathered}
{\left[h_{0}(\alpha)\right](t)=\lambda\left[\alpha(0), \omega_{1}\right](t)=\lambda[\alpha(0), \omega](t)=\lambda[\alpha(0), p(\alpha)](t)=[\tilde{\lambda}(\alpha)](t), \quad 0 \leq t \leq 1,} \\
{\left[h_{1}(\alpha)\right](t)=\alpha_{1}(t)=\alpha(t)=\left[i d_{E^{I}}(\alpha)\right](t), \quad 0 \leq t \leq 1, \quad \mathrm{e}} \\
p\left(h_{s}(\alpha(t))\right)=\left\{\begin{array}{l}
p\left(\alpha_{s}(t)\right)=p(\alpha(t)), \\
p\left(\left[\lambda\left(\alpha(s), \omega_{1-s}\right)\right](t-s)\right)=\omega_{1-s}(t-s), \quad s \leq t \leq 1,
\end{array}\right.
\end{gathered}
$$

como $s \leq t \leq 1 \Rightarrow 0 \leq t-s \leq 1-s$, segue que $\omega_{1-s}(t-s)=\omega(t)$, logo

$$
p\left(h_{s}(\alpha(t))\right)= \begin{cases}p(\alpha(t)), & 0 \leq t \leq s \\ \omega(t)=p(\alpha(t)), & s \leq t \leq 1\end{cases}
$$

Portanto, $h_{s}$ é a homotopia desejada.

Proposição 14. Seja $(E, p, B)$ um espaço fibrado com $B$ conexo, então para todo $b \in B$, as fibras $p^{-1}(b)$ possuem o mesmo tipo homotópico.

Demonstração. Sejam $b, b^{\prime} \in B, F=p^{-1}(b), F^{\prime}=p^{-1}\left(b^{\prime}\right), \omega$ um caminho de $b$ a $b^{\prime}$ e $\omega^{*}$ seu inverso, ou seja, $\omega^{*}(t)=\omega(1-t)$. Se $\lambda$ é a função levantamento de $(E, p, B)$, 
definimos $\phi: F \rightarrow F^{\prime}$ por

$$
\phi(x)=[\lambda(x, \omega)](1)
$$

e $\psi: F^{\prime} \rightarrow F$ por

$$
\psi\left(x^{\prime}\right)=\left[\lambda\left(x^{\prime}, \omega^{*}\right)\right](1) .
$$

Sejam $\tilde{\lambda}$ a aplicação do lema $5, h_{t}$ a homotopia conectando $\tilde{\lambda}$ e $i d_{E^{I}}$ preservando fibras, e $g_{t}: F \rightarrow F$ uma homotopia dada por

$$
g_{t}(x)=\left[h_{t}\left(\lambda(x, \omega)^{*}\right)\right](1)
$$

onde $\left[\lambda(x, \omega)^{*}\right](t)=[\lambda(x, \omega)](1-t)$. Então

$$
g_{0}(x)=\left[h_{0}\left(\lambda(x, \omega)^{*}\right)\right](1)
$$

seja $\alpha=\lambda(x, \omega)^{*}$, então

$$
\begin{gathered}
g_{0}(x)=\left[h_{0}(\alpha)\right](1)=[\lambda(\alpha(0), p(\alpha))](1)=\left[\lambda\left(\alpha(0), \omega^{*}\right)\right](1)=\left[\lambda\left(\left[\lambda(x, \omega)^{*}\right](0), \omega^{*}\right](1)=\right. \\
{\left[\lambda\left([\lambda(x, \omega)](1), \omega^{*}\right](1)=\psi(\phi(x)),\right.}
\end{gathered}
$$

e temos também que

$$
g_{1}(x)=\left[h_{1}(\alpha)\right](1)=\alpha(1)=\left[\lambda(x, \omega)^{*}\right](1)=[\lambda(x, \omega)](0)=x,
$$

e como $h_{t}$ preserva projeções, $g_{t}(x) \in F$, e portanto, $\psi \phi \sim 1$. De forma análoga, temos que $\phi \psi \sim 1$. Logo, $F$ e $F^{\prime}$ são homotópicamente equivalentes.

Definição 6. Seja $(E, p, B)$ um espaço fibrado fibrado, a fibra do espaço é dada por $F$, onde $F$ possui o mesmo tipo homotópico de $p^{-1}(b)$ para algum $b \in B$.

Definição 7. Uma aplicação $p: E \rightarrow B$ satisfaz a propriedade de levantamento de homotopias (PLH) para o espaço $X$ se, dada uma aplicação $g: X \rightarrow B$ e uma homotopia $f_{t}: X \rightarrow B$ tal que $f_{0}=p \circ g$, existe um levantamento da homotopia $f_{t}$, ou seja, existe uma homotopia $g_{t}: X \rightarrow E$ tal que $p \circ g_{t}=f_{t}$ e $g_{0}=g$, conforme ilustra o diagrama abaixo:

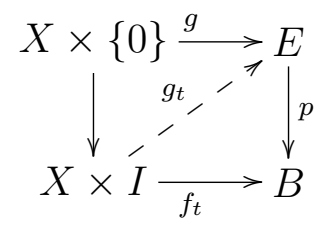

$A$ aplicação $p: E \rightarrow B$ satisfaz a propriedade de levantamento de homotopias 
absoluta (PLHA) se satisfaz PLH para todo espaço $X$.

A proposição a seguir mostra que a PLHA é equivalente a PLC.

Proposição 15 (Teorema de levantamento de homotopias para espaços fibrados). A terna $(E, p, B)$ é um espaço fibrado se, e somente se, $p: E \rightarrow B$ satisfaz a propriedade de levantamento de homotopias absoluta.

Demonstração. $(\Rightarrow)$ Sejam $g: X \rightarrow E$ uma aplicação e $f_{t}: X \rightarrow B$ uma homotopia tal que $f_{0}=p \circ g$.

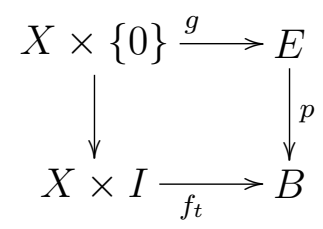

A partir de $f_{t}$ podemos construir uma aplicação $h: X \rightarrow B^{I}$ definida por

$$
[h(x)](t)=f_{t}(x), \quad x \in X, \quad t \in I .
$$

Sejam $k: X \rightarrow \Omega_{p}$ a aplicação definida por

$$
k(x)=(g(x), h(x)), \quad x \in X
$$

e $\lambda$ a função levantamento de $(E, p, B)$. Então a composta $\lambda \circ k$ é uma aplicação de $X$ em $E^{I}$. Podemos definir uma homotopia $g_{t}: X \rightarrow E$ dada por

$$
g_{t}(x)=[\lambda(k(x))](t), \quad x \in X, \quad t \in I .
$$

Portanto, segue que $g_{0}=g$ e $p \circ g_{t}=f_{t}, t \in I$.

$(\Leftarrow)$ Sejam $\eta_{0}: \Omega_{p} \rightarrow E$ a projeção na primeira coordenada e $\xi: \Omega_{p} \times I \rightarrow B$ uma homotopia dada por

$$
\xi((e, \omega), t)=\omega(t), \quad(e, \omega) \in \Omega_{p}, \quad t \in I .
$$

Temos então que $\xi((e, \omega), 0)=\omega(0)=p(e)=p\left(\eta_{0}(e, \omega)\right)$.

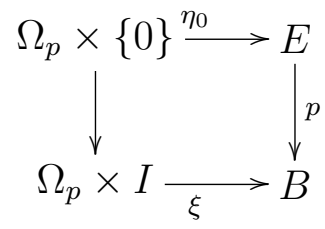

Por hipótese, existe uma homotopia $\eta: \Omega_{p} \times I \rightarrow E$ tal que $\eta((\omega, e), 0)=\eta_{0}(\omega, e)$, 
para todo $(\omega, e) \in \Omega_{p}$, e ainda, $p \circ \eta=\xi$.

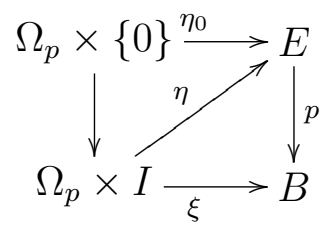

A partir de $\eta$ podemos construir uma aplicação $\Lambda: \Omega_{p} \rightarrow E^{I}$ definida por

$$
[\Lambda(z)](t)=\eta(z, t), \quad z \in \Omega_{p}, \quad t \in I
$$

Portanto, segue que $\tilde{p}(\Lambda(z))=z$, para todo $z \in \Omega_{p}$, logo, $(E, p, B)$ é um espaço fibrado.

Definição 8. A aplicação $p: E \rightarrow B$ satisfaz a propriedade da estrutura fatiada - PEF se existem uma cobertura aberta $\mathcal{U}=\{U\}$ de $B$ e um conjunto de aplicações $\left\{\phi_{U}: U \times p^{-1}(U) \subset B \times E \rightarrow E \mid U \in \mathcal{U}\right\}$ tais que

1. $p \circ \phi_{U}(b, x)=b$;

2. $\phi_{U}(p(x), x)=x$.

O resultado a seguir apresenta condições para que PEF implique PLHA, e portanto, PLC.

Proposição 16. Se a aplicação $p: E \rightarrow B$ satisfaz a propriedade da estrutura fatiada, e ainda, $E$ e $B$ são espaços métricos então $(E, p, B)$ é um espaço fibrado.

A demonstração do resultado anterior pode ser encontrada em [5].

Definição 9. Sejam $(E, p, B)$ um espaço fibrado e $\lambda$ sua função levantamento, $\lambda$ é regular se para todo $e \in E$ temos que $\lambda(e, p e)=e$, ou seja, caminhos degenerados em $B$ consistindo em um ponto são levantados para caminhos degenerados em E. A tripla $(E, p, B)$ é um espaço fibrado regular se ele admite uma função levantamento regular.

Proposição 17. Se B é um espaço métrico, então $(E, p, B)$ é um espaço fibrado regular.

Demonstração. Seja $d$ a métrica de $B$. Suponhamos que diâmetro de $B$ é menor ou igual a 1. Caso contrário, substituimos, sem perda de generalidade, a métrica de $B$ 
pela métrica $d /(1+d)$. Dado $\omega \in B^{I}$, sejam $d_{\omega}$ o diâmetro de $\omega(I), d_{\omega} \leq 1$, e $\omega^{\prime}$ o caminho dado por

$$
\omega^{\prime}(t)= \begin{cases}\omega\left(t / d_{\omega}\right), & 0 \leq t<d_{\omega} \\ \omega(1), & d_{\omega} \leq t \leq 1\end{cases}
$$

Se $\lambda$ é uma função levantamento de $(E, p, B)$, podemos obter uma função levantamento regular $\lambda^{\prime}: \Omega_{p} \rightarrow E^{I}$, onde $\lambda^{\prime}(e, \omega)$ é o caminho dada por

$$
\left[\lambda^{\prime}(e, \omega)\right](t)=\left[\lambda\left(e, \omega^{\prime}\right)\right]\left(d_{\omega} \cdot t\right)
$$

Para verificarmos que $\lambda^{\prime}$ é uma função levantamento regular, tomemos $(e, \alpha) \in \Omega_{p}$, onde $\alpha$ é o caminho degenerado que, para todo $t \in I$, assume o valor $p(e) \in B$. Como a imagem de $\alpha$ consiste em um único ponto, temos que $d_{\alpha}=0, \operatorname{logo}, \alpha^{\prime}(t)=\alpha(1)=e$, para todo $t \in I$. Portanto,

$$
\left[\lambda^{\prime}(e, \alpha)\right](t)=\left[\lambda\left(e, \alpha^{\prime}\right)\right]\left(d_{\alpha} \cdot t\right)=\left[\lambda\left(e, \alpha^{\prime}\right)\right](0)=e,
$$

para todo $t \in I$, ou seja, $\lambda^{\prime}(e, \alpha)$ é um caminho degenerado em $E^{I}$, logo, $\lambda^{\prime}$ é uma função levantamento regular, e $(E, p, B)$ é um espaço fibrado regular.

Proposição 18 (Teorema de extensão de homotopias para espaços fibrados). Sejam $(E, p, B)$ um espaço fibrado e $(X, A)$ um par de espaços métricos (A fechado em $X)$, tais que $E$ é $A N R$ ou $X$ e $A$ são ambos $A N R$ 's. Então para toda aplicação $g: X \rightarrow E$ e toda homotopia $h_{t}: A \rightarrow E$ tal que $h_{0}=g_{\downarrow A}$ e $p\left(h_{t}(x)\right)=p(g(x))$, $x \in A, t \in I$, pode ser estendida a uma homotopia $g_{t}: X \rightarrow E$ tal que $g_{0}=g$, $g_{t}(x)=h_{t}(x)$ para $x \in A$, e $p\left(g_{t}(x)\right)=p(g(x))$ para $x \in X, t \in I$.

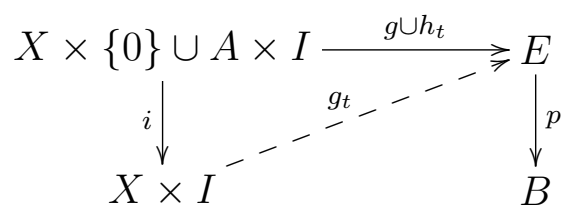

Demonstração. Como $E$ é $A N R$ ou $X$ e $A$ são ambos $A N R$ 's, pelas proposições 10 e 11 , temos que $A$ possui a $\mathrm{PEH}$ em $X$ com respeito a $E$. Logo, $g$ pode ser estendida a uma homotopia $g_{t}^{\prime}: X \rightarrow E$, mas que não necessariamente conserva as fibras. A partir de $g_{t}^{\prime}$, vamos construir a aplicação $G^{\prime}: X \rightarrow E^{I}$ dada por

$$
G^{\prime}(x)=\alpha \text {, onde } \alpha(t)=g_{t}^{\prime}(x) .
$$


Para $\alpha \in E^{I}, t \in I$, seja $\alpha^{t} \in E^{I}$ o caminho dado por

$$
\alpha^{t}(s)=\alpha(t \cdot(1-s)), \quad 0 \leq s \leq 1
$$

Então $\alpha^{0}(s)$ é o caminho constante que assume o valor $\alpha(0), \alpha^{1}(s)=\alpha(1-s)$, $\alpha^{t}(0)=\alpha(t)$ e $\alpha^{t}(1)=\alpha(0)$. Sejam $\lambda$ a função levantamento de $(E, p, B)$ e $g_{t}: X \rightarrow$ $E$ a homotopia dada por

$$
g_{t}(x)=\left[\lambda\left(\left[G^{\prime}(x)\right](t),\left(p\left(G^{\prime}(x)\right)\right)^{t}\right)\right](1)
$$

Temos então que $g_{0}(x)=\left[\lambda\left(\left[G^{\prime}(x)\right](0),\left(p\left(G^{\prime}(x)\right)\right)^{0}\right)\right](1)$. Sendo $B$ um espaço métrico, temos, pela proposição anterior, que $\lambda$ é uma função levantamento regular, e como $\left.\left(p\left(G^{\prime}(x)\right)\right)^{0}\right)$ é um caminho degenerado, que assume unicamente o valor $\left[\left(p\left(G^{\prime}(x)\right)\right)\right](0)$, temos que $\lambda\left(\left[G^{\prime}(x)\right](0),\left(p\left(G^{\prime}(x)\right)\right)^{0}\right)$ é o caminho degenerado que assume unicamente o valor $\left[G^{\prime}(x)\right](0)$, logo,

$$
g_{0}(x)=\left[G^{\prime}(x)\right](0)=g_{0}^{\prime}(x)=g(x)
$$

Temos ainda, que

$$
\begin{aligned}
& {\left[p\left(G^{\prime}(x)\right)^{t}\right](s)=\left[p\left(G^{\prime}(x)\right)\right](t \cdot(1-s))=} \\
& =p\left(\left[G^{\prime}(x)\right](t \cdot(1-s))\right)=p\left(g_{(t \cdot(1-s))}^{\prime}(x)\right) .
\end{aligned}
$$

Se $x \in A$, então $p\left(g_{t}^{\prime}(x)\right)=p\left(h_{t}(x)\right)=p(g(x))$, e portanto, para todo $x \in A$, temos que

$$
\left[p\left(G^{\prime}(x)\right)^{t}\right](s)=p\left(h_{(t \cdot(1-s))}(x)\right)=p(g(x))
$$

Agora, $p\left(G^{\prime}(x)\right)^{t}$ é um caminho degenerado, que assume unicamente o valor $p(g(x))$, e, novamente por regularidade, $\lambda\left(\left[G^{\prime}(x)\right](t),\left(p\left(G^{\prime}(x)\right)\right)^{t}\right)$ é o caminho degenerado que assume, portanto, unicamente o seu valor em $t=0$, e portanto, para todo $x \in A$,

$$
g_{t}(x)=\left[\lambda\left(\left[G^{\prime}(x)\right](t),\left(p\left(G^{\prime}(x)\right)\right)^{t}\right)\right](1)=\left[G^{\prime}(x)\right](t)=h_{t}(x) .
$$

Finalmente,

$$
\begin{gathered}
p\left(g_{t}(x)\right)=\left[p\left(\left(G^{\prime}(x)\right)^{t}\right)\right](1)= \\
p\left(\left[G^{\prime}(x)\right](0)\right)=p\left(g_{0}^{\prime}(x)\right)=p(g(x)) .
\end{gathered}
$$

Portanto, segue que $g_{t}$ é a homotopia desejada. 


\subsection{Par fibrado}

Definição 10. $\left(E_{0}, p_{0}, B\right)$ é um subespaço fibrado do espaço fibrado $(E, p, B)$ se, $E_{0} \subset E, p_{0}=p_{\rfloor E_{0}} e(E, p, B)$ admite uma função levantamento $\lambda$ tal que $\lambda\left(e_{0}, \omega\right) \in E_{0}^{I}$ se $e_{0} \in E_{0}$.

Definição 11. $\mathcal{F}=\left(E, E_{0}, p, B\right)$ é um par fibrado se $\left(E_{0}, p_{0}, B\right)$ é um subespaço fibrado de $(E, p, B)$.

Definição 12. Sejam $\mathcal{F}=\left(E, E_{0}, p, B\right)$ e $\mathcal{F}^{\prime}=\left(E^{\prime}, E_{0}^{\prime}, p^{\prime}, B\right)$ pares fibrados sobre a mesma base $B$. Uma aplicação de pares fibrados $\psi: \mathcal{F} \rightarrow \mathcal{F}^{\prime}$ é uma aplicação $\psi:\left(E, E_{0}\right) \rightarrow\left(E^{\prime}, E_{0}^{\prime}\right)$ tal que $p^{\prime} \circ \psi=p$, ou seja, $\psi$ preserva fibras.

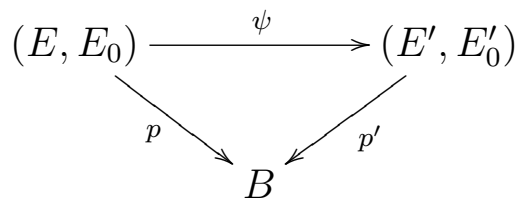

Seja $\phi: \mathcal{F} \rightarrow \mathcal{F}^{\prime}$ outra aplicação de pares fibrados, $\psi$ e $\phi$ são homotópicas preservando fibras se existe uma homotopia $h_{t}:\left(E, E_{0}\right) \rightarrow\left(E^{\prime}, E_{0}^{\prime}\right)$ tal que $h_{0}=\psi$, $h_{1}=\phi$ e $p^{\prime}\left(h_{t}(x)\right)=p(x), x \in E, t \in I$.

Definição 13. $\mathcal{F}$ e $\mathcal{F}^{\prime}$ possuem o mesmo tipo homotópico como espaços fibrados se existem aplicações de pares fibrados $\phi: \mathcal{F} \rightarrow \mathcal{F}^{\prime}$ e $\psi: \mathcal{F}^{\prime} \rightarrow \mathcal{F}$ tais que $\phi \psi \sim 1$ e $\psi \phi \sim 1$ preservando fibras, neste caso, $\phi e \psi$ são equivalências homotópicas fibradas.

No caso em que $E_{0}=\emptyset$, as duas últimas definições se reduzem a definições para espaços fibrados no lugar de pares fibrados.

Lema 6. Sejam $\mathcal{F}=\left(E, E_{0}, p, B\right)$ um par fibrado, $U \subset B$ contrátil em $B$ a um ponto $b_{0} \in U$, e $\mathcal{F}_{j U}=\left(p^{-1}(U), p_{0}^{-1}(U), p, U\right)$. Então $\mathcal{F}_{j U}$ e $\left(U \times F, U \times F_{0}, \pi, U\right)$ possuem o mesmo tipo homotópico como espaços fibrados, onde $F=p^{-1}\left(b_{0}\right), F_{0}=p_{0}^{-1}\left(b_{0}\right) e$ $\pi: U \times F \rightarrow U$ é a projeção na primeira coordenada.

Demonstração. Como $U \subset B$ é contrátil em $B$ a um ponto $b_{0} \in U$, existe uma aplicação $h: U \rightarrow B^{I}$ tal que $[h(b)](0)=b$ e $[h(b)](1)=b_{0}$. Sejam $\lambda$ a função levantamento do par fibrado, $\phi: p^{-1}(U) \rightarrow U \times F$ dada por

$$
\phi(x)=(p(x),[\lambda(x, h(p(x)))](1))
$$


e $\psi: U \times F \rightarrow p^{-1}(U)$ dada por

$$
\psi(b, f)=\left[\lambda\left(f, h(b)^{*}\right)\right](1)
$$

onde $h(b)^{*}$ é o inverso do caminho $h(b)$. Sejam $\tilde{\lambda}$ a aplicação do lema $5, h_{t}$ a homotopia conectando $\tilde{\lambda}$ e $i d_{E^{I}}$ preservando fibras, e $g_{t}: p^{-1}(U) \rightarrow p^{-1}(U)$ uma homotopia dada por

$$
g_{t}(x)=\left[h_{t}\left(\lambda(x, h(p(x)))^{*}\right)\right](1) .
$$

Então,

$$
g_{0}(x)=\left[h_{0}\left(\lambda(x, h(p(x)))^{*}\right)\right](1),
$$

seja $\alpha=\lambda(x, h(p(x)))^{*}$, então

$$
g_{0}(x)=\left[h_{0}(\alpha)\right](1)=[\lambda(\alpha(0), p(\alpha))](1),
$$

como

$$
\alpha(0)=\left[\lambda(x, h(p(x)))^{*}\right](0)=[\lambda(x, h(p(x))](1),
$$

$\mathrm{e}$

$$
p(\alpha)=p\left(\lambda(x, h(p(x)))^{*}\right)=h(p(x))^{*},
$$

temos que,

$$
g_{0}(x)=\left[\lambda\left([\lambda(x, h(p(x)))](1), h(p(x))^{*}\right)\right](1)=(\psi \circ \phi)(x),
$$

e temos também que,

$$
g_{1}(x)=\left[h_{1}\left(\lambda(x, h(p(x)))^{*}\right](1)=\left[\left(\lambda(x, h(p(x)))^{*}\right](1)=[(\lambda(x, h(p(x)))](0)=x,\right.\right.
$$

e portanto, $\psi \phi \sim 1$, e como $h_{t}$ preserva projeções, $g_{t}$ também preserva projeções,. De forma análoga, temos que $\phi \psi \sim 1$ preservando projeções. Logo, $\left(p^{-1}(U), p_{0}^{-1}(U), p, U\right)$ e $\left(U \times F, U \times F_{0}, \pi, U\right)$ possuem o mesmo tipo homotópico como espaços fibrados.

\subsection{Fibrado vetorial generalizado e principais re- sultados}

Definição 14. ( $E, p, B)$ é um fibrado vetorial generalizado (fvg) se existe uma seção $s: B \rightarrow E$ tal que, se $E_{0}=E-s(B)$ então; 
1. $\mathcal{F}=\left(E, E_{0}, p, B\right)$ é um par fibrado com fibra $\left(F, F_{0}\right)$;

2. existe uma homotopia $h_{t}: F \rightarrow F$ tal que $h_{t}\left(F_{0}\right) \subset F_{0}$ se $t \in\left[0,1\left[\right.\right.$, e $h_{1}(F)=$ $F \cap s(B)$.

$(E, p, B)$ é um n-fibrado vetorial generalizado (n-fvg), sendo $n \geq 2$, se é um fvg $e$ :

1. $F_{0}$ é conexo por caminhos e, quando $n \geq 3, \pi_{1}\left(F_{0}\right)=0$;

2. $H_{*}\left(F, F_{0}\right) \cong H_{*}\left(E^{n}, E^{n}-0\right)$.

Se $\sigma: B \rightarrow E$ é uma outra seção em $\mathcal{F}$, dizemos que $b \in B$ é uma singularidade de $\sigma$ se $\sigma(b)=s(b)$.

Lema 7. Se $\mathcal{F}=\left(E, E_{0}, p, B\right)$ é um par fibrado e $f: X \rightarrow B$ é uma aplicação, então $f^{*}(\mathcal{F})=\left(f^{*}(E), f^{*}\left(E_{0}\right), p^{*}, X\right)$ é um par fibrado induzido por $f$, onde

$$
\begin{aligned}
& f^{*}(E)=\{(x, e) \in X \times E \mid f(x)=p(e)\}, \\
& f^{*}\left(E_{0}\right)=\left\{(x, e) \in X \times E_{0} \mid f(x)=p_{0}(e)\right\} \text {, } \\
& p^{*}(x, e)=x,
\end{aligned}
$$

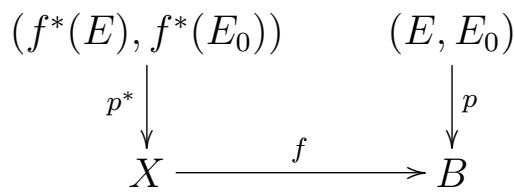

e se $\mathcal{F}$ é um $n$-fvg, então $f^{*}(\mathcal{F})$ também é um $n$-fvg.

Demonstração. Seja $\lambda$ a função levantamento do par fibrado $\mathcal{F}$. Seja $\Omega_{p^{*}}=\left\{\left(e^{*}, \omega\right) \in\right.$ $\left.f^{*}(E) \times X^{I} \mid p^{*}\left(e^{*}\right)=\omega(0)\right\}$. Dados $e^{*}=(\omega(0), e) \in f^{*}(E)$ e $\omega \in X^{I}$, seja $\lambda^{*}: \Omega_{p^{*}} \rightarrow\left(f^{*}(E)\right)^{I}$ dada por

$$
\lambda^{*}((\omega(0), e), \omega)=(\omega, \lambda(e, f(\omega))) .
$$

Temos que $\omega \in X^{I}$, como $\lambda$ é uma função levantamento de $\mathcal{F}$ temos que $\lambda(e, f(\omega)) \in$ $E^{I}$, e além disso $p(\lambda(e, f(\omega)))=f(\omega), \operatorname{logo},(\omega, \lambda(e, f(\omega))) \in\left(f^{*}(E)\right)^{I}$, e portanto, $\lambda^{*}$ é uma função levantamento e $\left(f^{*}(E), p^{*}, X\right)$ é um espaço fibrado. Se $(\omega(0), e) \in$ $f^{*}\left(E_{0}\right)$, temos que $e \in E_{0}$, e como $\mathcal{F}$ é um par fibrado e $\lambda$ é uma função levantamento de $\mathcal{F}$, segue que $\lambda(e, f(\omega)) \in E_{0}^{I}$, e como $\omega \in X^{I}$, temos que $(\omega, \lambda(e, f(\omega))) \in$ $f^{*}\left(E_{0}\right)^{I}$, portanto, $\left(f^{*}\left(E_{0}\right), p^{*}, X\right)$ é um subespaço fibrado de $\left(f^{*}(E), p^{*}, X\right)$ e $f^{*}(\mathcal{F})$ é um par fibrado. 
Lema 8. Sejam $\mathcal{F}=\left(E, E_{0}, p, B\right)$ um par fibrado e $X$ um espaço que domina $B$ por meio das aplicações $f: X \rightarrow B$ e $g: B \rightarrow X$. O espaço fibrado induzido $f^{*}(\mathcal{F})=\left(f^{*}(E), f^{*}\left(E_{0}\right), p^{*}, X\right)$ admite uma seção $\sigma^{*}$ se, e somente se, $\mathcal{F}$ admite uma seção $\sigma$. Se $\sigma^{*}$ é uma seção em $f^{*}(\mathcal{F})$ e $x \in X \operatorname{com}^{*}(x) \in f^{*}\left(E_{0}\right)$, então para uma seção $\sigma$ em $\mathcal{F}$ obtida de $\sigma^{*}$ temos que se $b \in B$ com $g(b)=x$, então $\sigma(b) \in E_{0}$.

Demonstração. $(\Leftarrow)$ Se existe uma seção $\sigma: B \rightarrow E$, definimos $\sigma^{*}: X \rightarrow f^{*}(E)$ por $\sigma^{*}(x)=(x, \sigma f(x))$.

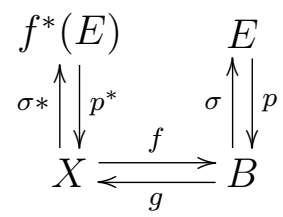

$(\Rightarrow)$ Dado $\sigma^{*}$, definimos $f^{-}(x, e)=e$, assim temos

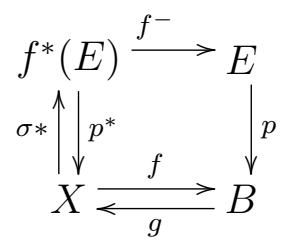

onde $p^{*} \sigma^{*}=i d$. Seja $h_{t}: B \rightarrow B$ a homotopia tal que $h_{0}=f \circ g$ e $h_{1}=i d_{B}$. Definimos $\sigma^{\prime}: B \rightarrow E$ por $\sigma^{\prime}=f^{-} \sigma^{*} g$, e então $p \circ \sigma^{\prime}=f \circ g=h_{0}$. Portanto, pela proposição 15 existe um levantamento $g_{t}$ da homotopia $h_{t}$, tal que $g_{0}=\sigma^{\prime}$ e $p \circ g_{1}=h_{1}=i d_{B}$, logo, $\sigma=g_{1}$ é a seção desejada de $\mathcal{F}$. A segunda parte do teorema segue facilmente da proposição 15 e do fato de que $f^{-}\left(f^{*}\left(E_{0}\right)\right) \subset E_{0}$.

Lema 9. Sejam $\mathcal{F}=\left(E, E_{0}, p, B\right)$ um fvg sobre o espaço métrico $B$ e $U \subset B$ contrátil em $B$ a um ponto $b_{0} \in U$ tal que $b_{0}$ se mantém fixo durante a contração. Então existe uma retração por deformação preservando fibras $r_{t}: p^{-1}(U) \rightarrow p^{-1}(U)$ tal que $r_{t}\left(p_{0}^{-1}(U)\right) \subset E_{0}$ para $t \in\left[0,1\left[, r_{1}\left(p^{-1}(U)\right) \subset s(B)\right.\right.$ e $s\left(b_{0}\right)$ se mantém fixo durante a retração.

Demonstração. Sejam $\bar{\lambda}: E^{I} \rightarrow E^{I}$ a aplicação do lema 5 dada por $\bar{\lambda}(\alpha)=$ $\lambda(\alpha(0), p(\alpha))$ e $h_{t}: E^{I} \rightarrow E^{I}$ a homotopia conectando $\bar{\lambda}$ e $i d_{E^{I}}$ preservando fibras, pela demonstração do lema, vemos que se $\alpha(r) \in E_{0}$ então $h_{t}(\alpha(r)) \in E_{0}$ para $t \in[0,1[$. Sejam

$$
\left(p^{-1}(U), p_{0}^{-1}(U)\right) \underset{\psi}{\stackrel{\phi}{\gtrless}}\left(U \times F, U \times F_{0}\right)
$$

as equivalências do lema 6 induzidas pela contração de $U$, e $g_{t}:\left(p^{-1}(U), p_{0}^{-1}(U)\right) \rightarrow$ $\left(p^{-1}(U), p_{0}^{-1}(U)\right)$ a homotopia preservando fibras tal que $g_{0}(e)=e$ e $g_{1}(e)=\psi \circ \phi(e)$. 
Como $\mathcal{F}$ é um fvg, existe uma homotopia $f_{t}: F \rightarrow F$ tal que $f_{0}(e)=e, f_{1}(e)=s\left(b_{0}\right)$ e $f_{t}\left(F_{0}\right) \subset F_{0}$ para $t \in\left[0,1\left[\right.\right.$. Seja $c_{t}: U \rightarrow B$ a contração de $U$ em $b_{0}$ que mantém $b_{0}$ fixo. Para $b \in U$, definamos $\omega_{b} \in B^{I}$ por $\omega_{b}(t)=c_{1-t}(b)$, então $\psi\left(b, s\left(b_{0}\right)\right)=$ $\left[\lambda\left(s\left(b_{0}\right), \omega_{b}\right)\right](1)$. Definamos agora, a retração por deformação $r_{t}$ de $p^{-1}(U)$ em $s(U)$ por

$$
r_{t}(e)=\left\{\begin{array}{lr}
g_{3 t}(e), & 0 \leq t \leq 1 / 3, \\
\psi\left(p(e), f_{3 t-1}\left(\pi_{2} \circ \phi(e)\right)\right), & 1 / 3 \leq t \leq 2 / 3, \\
{\left[h_{3 t-2}\left(s\left(\omega_{p(e)}\right)\right)\right](1),} & 2 / 3 \leq t \leq 1,
\end{array}\right.
$$

onde $\pi_{1}: U \times F \rightarrow U$ é a projeção na primeira coordenada e $\pi_{2}: U \times F \rightarrow F$ é a projeção na segunda coordenada. Para $t=1 / 3$, temos que

$$
\begin{aligned}
g_{1}(e) & =\psi(\phi(e)) \\
& =\psi\left(\pi_{1} \circ \phi(e), \pi_{2} \circ \phi(e)\right) \\
& =\psi\left(p(e), i d \circ \pi_{2} \circ \phi(e)\right) \\
& =\psi\left(p(e), f_{0}\left(\pi_{2} \circ \phi(e)\right)\right),
\end{aligned}
$$

e para $t=2 / 3$

$$
\begin{aligned}
\psi\left(p(e), f_{1}\left(\pi_{2} \circ \phi(e)\right)\right) & =\psi\left(p(e), s\left(b_{0}\right)\right), \text { pois } f_{1}=s\left(b_{0}\right) \\
& \left.=\left[\lambda\left(s\left(b_{0}\right), \omega_{p(e)}\right)\right)\right](1) \\
& =\left[(\lambda \circ \tilde{p})\left(s\left(\omega_{p(e)}\right)\right)\right](1) \\
& =\left[\bar{\lambda}\left(s\left(\omega_{p(e)}\right)\right)\right](1) \\
& =\left[h_{0}\left(s\left(\omega_{p(e)}\right)\right)\right](1),
\end{aligned}
$$

portanto, segue que $r_{t}$ é contínua. Além disso,

$$
r_{0}(e)=g_{0}(e)=i d_{p^{-1}(U)}(e)
$$

e também

$$
\begin{aligned}
r_{1}(e) & =\left[h_{1}\left(s\left(\omega_{p(e)}\right)\right)\right]((1) \\
& =\left[i d\left(s\left(\omega_{p(e)}\right)\right)\right](1) \\
& =\left[s\left(\omega_{p(e)}\right)\right](1) \\
& =s(p(e)) \in s(B),
\end{aligned}
$$

logo, $r_{t}$ é de fato uma deformação de $p^{-1}(U)$ em $s(U)$. Vamos agora analisar $r_{t}\left(s\left(b_{0}\right)\right)$. Para $0 \leq t \leq 1 / 3$, temos que

$$
r_{t}\left(s\left(b_{0}\right)\right)=g_{3 t}\left(s\left(b_{0}\right)\right)=\left[h_{3 t}\left(\lambda\left(s\left(b_{0}\right), \omega_{p\left(s\left(b_{0}\right)\right)}\right)^{*}\right)\right](1)=\left[h_{3 t}\left(\lambda\left(s\left(b_{0}\right), \omega_{b_{0}}\right)^{*}\right)\right](1) .
$$


Como $B$ é um espaço métrico, podemos tomar $\lambda$ regular, logo, $\lambda\left(s\left(b_{0}\right), \omega_{b_{0}}\right)$ é um caminho constante da forma $\alpha(r)=s\left(b_{0}\right)$ para $0 \leq r \leq 1$, portanto,

$$
r_{t}\left(s\left(b_{0}\right)\right)=\left[h_{3 t}(\alpha)\right](1)=[\lambda(\alpha(3 t), p(\alpha))](1-3 t)=\alpha(1-3 t)=s\left(b_{0}\right) .
$$

Para $1 / 3 \leq t \leq 2 / 3$, temos que

$$
r_{t}\left(s\left(b_{0}\right)\right)=\psi\left(p\left(s\left(b_{0}\right)\right), f_{3 t-1}\left(\pi_{2} \circ \phi\left(s\left(b_{0}\right)\right)\right)\right)=\psi\left(b_{0}, f_{3 t-1}\left(\left[\lambda\left(s\left(b_{0}\right), \omega_{b_{0}}\right)\right](1)\right)\right),
$$

pela regularidade de $\lambda$, obtemos

$$
r_{t}\left(s\left(b_{0}\right)\right)=\psi\left(b_{0}, f_{3 t-1}\left(s\left(b_{0}\right)\right)\right)=\left[\lambda\left(f_{3 t-1}\left(s\left(b_{0}\right)\right), \omega_{b_{0}}\right)\right](1)=\alpha(1)=s\left(b_{0}\right) .
$$

Para $2 / 3 \leq t \leq 1$, temos que

$$
r_{t}\left(s\left(b_{0}\right)\right)=\left[h_{3 t-2}\left(s\left(\omega_{p\left(s\left(b_{0}\right)\right)}\right)\right)\right](1)=\left[h_{3 t-2}\left(s\left(\omega_{b_{0}}\right)\right)\right](1)=\left[h_{3 t-2}(\alpha)\right](1),
$$

novamenta, pela regularidade de $\lambda$, obtemos

$$
r_{t}\left(s\left(b_{0}\right)\right)=[\lambda(\alpha(3 t-2), p(\alpha))](1-(3 t-2))=\alpha\left(1-(3 t-2)=s\left(b_{0}\right) .\right.
$$

E portanto, $r_{t}\left(s\left(b_{0}\right)\right)=s\left(b_{0}\right)$, e o ponto $s\left(b_{0}\right)$ se mantém fixo durante a retração por deformação.

Lema 10. Sejam $\mathcal{F}=\left(E, E_{0}, p, B\right)$ um fvg, $h:[-1,1]^{n} \rightarrow B$ um homeomorfismo, $C=h\left([-1,1]^{n}\right)$ e $h(0)=b \in B$. Se existe uma seção $\sigma^{\prime}: \partial C \rightarrow E_{0}$ então existe uma seção $\sigma: C \rightarrow E$ tal que $\sigma(C-b) \subset E_{0}$.

Demonstração. Consideremos a homotopia dada por $h_{t}(x)=h\left((1-t) \cdot h^{-1}(x)\right)$, temos então o seguinte diagrama

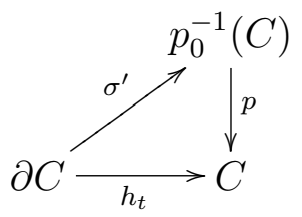

como $\sigma^{\prime}$ é uma seção temos que, para $t=0$, o diagrama é comutativo. Como $\left(p^{-1}(C), p_{0}^{-1}(C), p, C\right)$ é um par fibrado, a homotopia do diagrama possui um le- 
vantamento, a homotopia $\sigma_{t}: \partial C \rightarrow p_{0}^{-1}(C)$

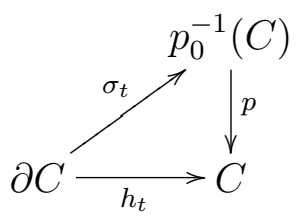

onde $p \circ \sigma_{t}=h_{t}$ e $\sigma_{0}=\sigma^{\prime}$. Sejam $s: B \rightarrow E$ a seção tal que $E_{0}=E-s(B)$ e $r_{t}: p^{-1}(C) \rightarrow p^{-1}(C)$ a retração por deformação do lema 9 , temos que $r_{t}\left(p_{0}^{-1}(C)\right) \subset$ $p_{0}^{-1}(C)$ para $t \in\left[0,1\left[\right.\right.$, e $r_{1}\left(p^{-1}(C)\right) \subset s(C)$.

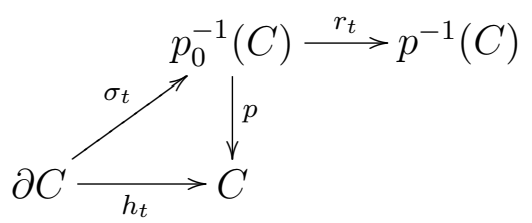

Sejam $F: \partial C \times I \rightarrow p^{-1}(C)$ a homotopia dada por $F(x, t)=r_{t}\left(\sigma_{t}(x)\right)$ e $H$ : $\partial C \times I \rightarrow C$ a homotopia dada por $H(x, t)=h_{t}(x)$. Notemos que, para todo elemento da forma $(c, 1) \in \partial C \times I$, temos que $H(c, 1)=h_{1}(c)=h(0)=b$, e ainda, como $p\left(\sigma_{1}(c)\right)=h_{1}(c)=h(0)=b$, logo, $\sigma_{1}(c) \in p^{-1}(b)$, e portanto, $F(c, 1)=$ $r_{1}\left(\sigma_{1}(c)\right)=s(b)$. Consideremos então, o espaço quociente $\partial C \times I / \partial C \times\{1\}$ munido com a topologia quociente. Como $\left(c_{1}, t_{1}\right) \sim\left(c_{2}, t_{2}\right)$ se, e somente se, $\left(c_{1}, t_{1}\right)=\left(c_{2}, t_{2}\right)$ ou $t_{1}=t_{2}=1$, temos que as aplicações induzidas dadas por

$$
\bar{F}:(c, t) \in \frac{\partial C \times I}{\partial C \times\{1\}} \mapsto F(c, t) \in p^{-1}(C)
$$

$\mathrm{e}$

$$
\bar{H}:(c, t) \in \frac{\partial C \times I}{\partial C \times\{1\}} \mapsto H(c, t) \in C
$$

estão bem definidas.

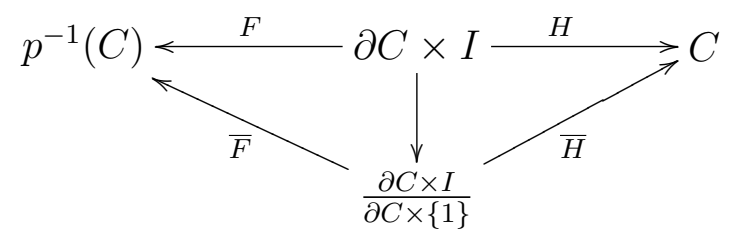

Como $H$ e $F$ são contínuas e $\partial C \times I / \partial C \times\{1\}$ possui a topologia quociente, temos que $\bar{H}$ e $\bar{F}$ são contínuas. Como $H$ é sobrejetora, temos que $\bar{H}$ é sobrejetora. Se $\bar{H}\left(c_{1}, t_{1}\right)=\bar{H}\left(c_{2}, t_{2}\right)$, temos que $h\left(\left(1-t_{1}\right) \cdot h^{-1}\left(c_{1}\right)\right)=h\left(\left(1-t_{2}\right) \cdot h^{-1}\left(c_{2}\right)\right)$, logo, $\left(1-t_{1}\right) \cdot h^{-1}\left(c_{1}\right)=\left(1-t_{2}\right) \cdot h^{-1}\left(c_{2}\right)$, e portanto, ou $t_{1}=t_{2}=1$ ou $\left(t_{1}, c_{1}\right)=\left(t_{2}, c_{2}\right)$, 
logo, segue que $\bar{H}$ é injetora. Então, temos que $\bar{H}$ é uma função contínua, bijetora, definida num compacto e assumindo valores em $C$ que é Hausdorff, portanto, $\bar{H}^{-1}$ existe e é uma função contínua. Portanto, podemos tomar $\sigma=\bar{F} \circ \bar{H}^{-1}$ que é a seção desejada. Para verificarmos que $\sigma$ estende $\sigma^{\prime}$, notemos que se $x \in \partial C$, então $\bar{H}^{-1}(x)=(x, 0), \log \mathrm{o}$,

$$
\sigma(x)=\bar{F}(x, 0)=r_{0}\left(\sigma_{0}(x)\right)=\sigma_{0}(x)=\sigma^{\prime}(x) .
$$

Lema 11. Sejam $\mathcal{F}=\left(E, E_{0}, p, P\right)$ um $n$-fvg com fibra $\left(F, F_{0}\right)$, onde $P$ é um poliedro n-dimensional conexo finito. Dados L, um sub-poliedro de $P$, e uma seção $\sigma$ em $\left(p_{0}^{-1}(L), p_{0}, L\right)$, podemos estender $\sigma$ a uma seção em $\mathcal{F}$ com um número finito de singularidades, todas contidas no interior de simplexos maximais.

Demonstração. Sejam $K$ a união dos simplexos contidos em $P-L$ e $K^{m}$ o m-ésimo esqueleto de $K$. Podemos estender $\sigma$ a $L \cup K^{0}$ associando um vértice $v$ a qualquer ponto de $p_{0}^{-1}(v)$. Suponhamos que $\sigma$ tenha sido estendida a $L \cup K^{m}, m<n-1$. Seja $c$ um $(\mathrm{m}+1)$-simplexo de $K$, então $\sigma$ está definida em $\partial c$ que é uma m-esfera topológica. Sejam

$$
p_{0}^{-1}(c) \underset{\psi}{\stackrel{\phi}{\gtrless}} c \times F_{0}
$$

equivalências homotópicas fibradas e $\pi_{2}: c \times F_{0} \rightarrow F_{0}$ a projeção na segunda coordenada. Então $\pi_{2} \phi \sigma: \partial c \rightarrow F_{0}$ aplicam uma m-esfera, $m<n-1$, em $F_{0}$ que é (n - 2)-conexo então $\pi_{2} \phi \sigma$ pode ser estendida a uma aplicação $\Sigma^{\prime}: c \rightarrow F_{0}$. Definimos $\Sigma: c \rightarrow p_{o}^{-1}(c)$ fazendo $\Sigma(x)=\psi\left(x, \Sigma^{\prime}(x)\right)$. Notemos que se $x \in \partial c$, então $\Sigma^{\prime}(x)=\pi_{2} \phi \sigma(x)$, logo, $\phi \sigma(x)=\left(x, \Sigma^{\prime}(x)\right)$ e $\Sigma(x)=\psi \phi \sigma(x)$, e portanto a homotopia que preserva fibras levando $\psi \phi$ na identidade induz uma homotopia preservando fibras $h_{t}^{\prime}: \partial c \rightarrow p_{0}^{-1}(\partial c)$ tal que $h_{0}^{\prime}(x)=\psi \phi \sigma(x)$ e $h_{1}^{\prime}(x)=\sigma(x)$. Sejam $h: c \rightarrow p_{o}^{-1}(c)$ tal que $h=\Sigma$, e $\left(h_{\jmath \partial c}\right)_{t}: \partial c \rightarrow p_{o}^{-1}(c)$ uma homotopia de $h_{\jmath \partial c}$ tal que $\left(h_{\jmath \partial c}\right)_{t}=h_{t}^{\prime}$, e portanto uma homotopia preservando fibras. Pelo teorema de extensão de homotopias para espaços fibrados, $\left(h_{\lrcorner \partial c}\right)_{t}$ pode ser estendida a $h_{t}: c \rightarrow p_{o}^{-1}(c)$ uma homotopia de $h$ que preserva fibras. Fazendo $\sigma(x)=h_{1}(x)$ podemos estender $\sigma$ a $c$. Repetindo a mesma construção em cada $(\mathrm{m}+1)$-simplexo de $K$, podemos estender $\sigma$ a $L \cup K^{m+1}$. Quando $\sigma$ for estendida a $L \cup K^{n-1}$, aplicamos o lema anterior em cada n-simplexo de $K$ e obtemos assim uma seção em $\mathcal{F}$ com no máximo uma singularidade para cada um desse n-simplexos, como $P$ é finito, $K$ também é finito, e portanto, a seção obtida terá um número finito de singularidades. 
Os resultados apresentados a partir deste ponto são estabelecidos para espaços fibrados onde o espaço base $B$ é uma n-variedade. Trataremos apenas os casos em que $n$ é maior que um, ou seja, as variedades consideradas são de dimensão ao menos dois.

Lema 12. Seja $\mathcal{F}=\left(E, E_{0}, p, B\right)$ um fvg onde $B$ é uma n-variedade. Se existe uma seção $\sigma^{\prime}$ de $\mathcal{F}$ com um número finito de singularidades, então existe uma seção $\sigma$ com apenas uma singularidade.

Demonstração. Sejam $x_{1}, \ldots, x_{q}$ as singularidades de $\sigma^{\prime}$. Pelo lema 4, existe uma célula topológica fechada $C$ contendo $x_{1}, \ldots, x_{q}$ em seu interior, ou seja, $x_{1}, \ldots, x_{q}$ estão contidas numa vizinhança euclidiana. Pelo lema 10, existe uma seção $\sigma \mathrm{em}$ $C$ tal que $\sigma_{\downarrow \partial C}=\sigma_{\jmath \partial C}^{\prime}$ e $\sigma(z) \in E_{0}$ exceto em um ponto. Fazendo $\sigma$ igual a $\sigma^{\prime}$ em $B-C$ estendemos a seção a todo $B$.

Teorema 3. Se $\mathcal{F}=\left(E, E_{0}, p, B\right)$ é um $n$-fvg com fibra $\left(F, F_{0}\right)$ e B é uma $n$ variedade compacta, então existe uma seção $\sigma$ de $\mathcal{F}$ com apenas uma singularidade.

Demonstração. Como B é uma n-variedade topológica compacta, consideremos uma cobertura por abertos $\mathcal{U}=\left\{U_{i}\right\}_{i \leq n}$ de $B$ formada por vizinhanças euclidianas. A variedade $B$ é compacta, logo, é um compacto ANR n-dimensional, pela proposição 2 , temos que se $P$ é o nervo da cobertura $\mathcal{U}$ então $P$ é um poliedro n-dimensional finito que $\epsilon$-domina $B$ por meio das aplicações $f: P \rightarrow B$ e $g: B \rightarrow P$,

$$
P \underset{f}{\stackrel{g}{\leftrightarrows}} B, \quad \text { com } \quad f \circ g \sim_{\epsilon} i b_{B}
$$

onde $g$ é uma aplicação baricêntrica dada por

$$
g(y)=\sum_{i=1}^{n} t_{i}(y) \cdot p_{i}, \quad \text { onde } \quad t_{i}(y)=\frac{d\left(y, B-U_{i}\right)}{\sum_{i=1}^{n} d\left(y, B-U_{i}\right)},
$$

conforme observado anteriormente, temos que $\sum_{i=1}^{n} d\left(y, X-U_{i}\right)>0$ e para todo $y \in B, \sum_{i=1}^{n} t_{i}(y)=1$. Pelo lema 7 , a aplicação $f: P \rightarrow B$ induz um n-fvg $f^{*}(\mathcal{F})=\left(f^{*}(E), f^{*}\left(E_{0}\right), p^{*}, P\right)$.

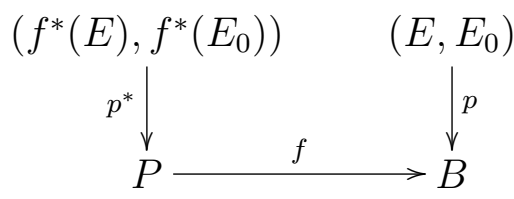


Consideremos um vértice $v$ de $P$ e uma função que associa este vértice a um elemento de $p_{0}^{*-1}(\{v\})$, temos portanto, uma seção em $\left(p_{0}^{*-1}(\{v\}), p_{0}^{*},\{v\}\right)$, e $\{v\}$ é um subpoliedro de $P$, logo, pelo lema 11 , esta seção pode ser estendida a uma seção $\sigma^{*}$ em $f^{*}(\mathcal{F})$ de modo que o conjunto de singularidades de $\sigma^{*}$ é finito. Sejam $\left\{x_{i}\right\}$ estas singularidades. Seja $s: B \rightarrow E$ a seção tal que $E_{0}=E-s(B)$. Pelo lema 8, como $f^{*}(\mathcal{F})$ admite uma seção $\sigma^{*}$, temos que $\mathcal{F}$ admite uma seção $\sigma^{\prime}$, e além disso, $\sigma^{\prime}(B) \cap s(B)=s\left(\cup g^{-1}\left(x_{i}\right)\right)$.

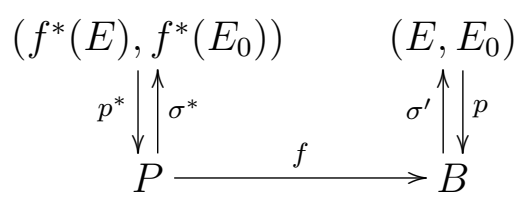

Pelo lema 11, temos que cada singularidade de $\sigma^{*}$ está no interior de um n-simplexo de $P$, consideremos então o n-simplexo $\left[p_{1}, \ldots, p_{n}\right]$ que contém a singularidade $x_{i}$ em seu interior, se $y \in g^{-1}\left(x_{i}\right)$, ou seja, $x_{i}=g(y)$, temos que $x_{i}=t_{1}(y) \cdot p_{1}+\ldots+t_{n}(y) \cdot p_{n}$, e como $x_{i}$ está no interior do simplexo $\left[p_{1}, \ldots, p_{n}\right]$, temos que $t_{1}(y), \ldots, t_{n}(y)>0$, em particular, $t_{1}(y)=d\left(y, B-U_{1}\right)>0$, logo, $y \in$ Int $U_{1}$, e portanto, $g^{-1}\left(x_{i}\right) \subset$ Int $U_{1}$. Seja $C \subset U_{1}$ uma célula topológica fechada contendo $g^{-1}\left(x_{1}\right)$ em seu interior. Sejam $d^{\prime}$ a distância, na métrica de $B$, de $g^{-1}\left(x_{1}\right)$ a $g^{-1}\left(x_{2}\right) \cup \ldots \cup g^{-1}\left(x_{n}\right)$ e $d^{\prime \prime}$ a distância de $g^{-1}\left(x_{1}\right)$ a $\partial C$, como $g^{-1}\left(x_{1}\right), \cup_{i=2}^{n} g^{-1}\left(x_{i}\right)$ e $\partial C$ são compactos, temos que $d^{\prime}$ e $d^{\prime \prime \prime}$ estão bem definidas. Seja $d=\min \left\{d^{\prime}, d^{\prime \prime}\right\}$. Tomemos uma triangulação finita de $C$ de norma menor que $d / 3$, então um simplexo desta triangulação que intersecta $g^{-1}\left(x_{1}\right)$ é disjunto de um simplexo que intersecta $g^{-1}\left(x_{2}\right) \cup \ldots \cup g^{-1}\left(x_{n}\right) \cup \partial C$. Seja $Q$ o

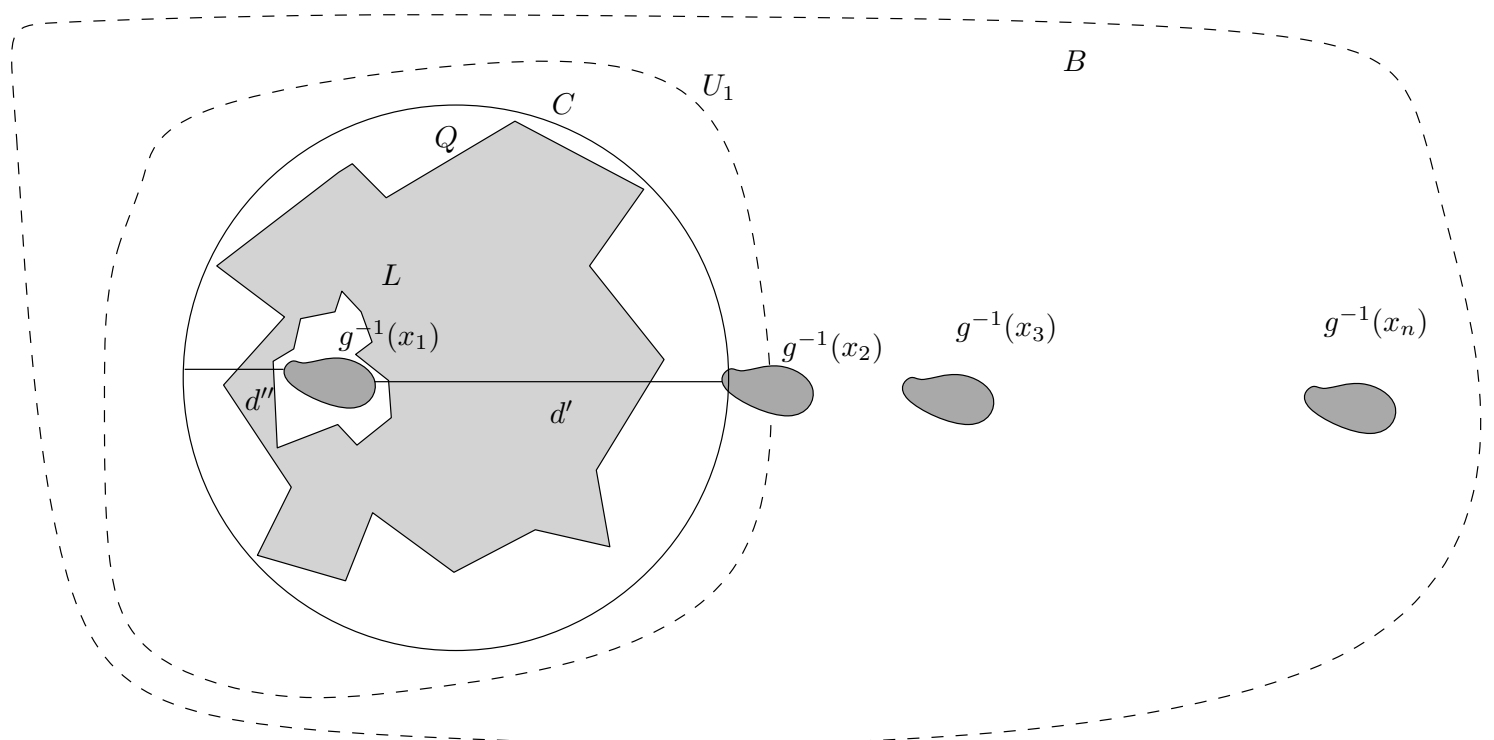

subpoliedro de $C$ consistindo de simplexos que não intersectam $\cup_{i=2}^{n} g^{-1}\left(x_{i}\right)$ e seja $L$ 
o subpoliedro de $Q$ consistindo de simplexos que não intersectam $g^{-1}\left(x_{1}\right)$. Notemos que $\sigma_{\lrcorner L}^{\prime}$ é uma seção em $\left(p_{0}^{-1}(L), p_{0}, L\right)$. O poliedro $Q$ não precisa ser conexo, mas $B$ é conexo por caminhos, $\log p_{0}^{-1}(b)$ é independente de $b \in B$ a menos de tipo homotópico, e como a conexidade de $Q$ no lema 11 é necessária somente para garantir que a pré-imagem de todo ponto de $Q$ sobre $p_{0}$ (indiscriminadamente chamado de $F_{0}$ ) possuisse o mesmo tipo homotópico, podemos aplicar o lema novamente. Logo, pelo lema 11, temos uma seção $\Sigma^{\prime}$ em $\left(p^{-1}(Q), p, Q\right)$, que coincide com $\sigma^{\prime}$ em $L$ e possui apenas um número finito de singularidades $\left\{b_{j}^{1}\right\}$. Fazendo $\Sigma^{\prime}$ igual a $\sigma^{\prime}$ em $B-Q$, obtemos uma extensão de $\sigma^{\prime}$ em $B$, e as singularidades de $\Sigma^{\prime}$ são $\left\{b_{j}^{1}\right\} \cup$ $g^{-1}\left(x_{2}\right) \cup \ldots \cup g^{-1}\left(x_{n}\right)$. Repetindo esta construção para $i>1$, obteremos, em um número finito de passos, uma seção $\Sigma$ de $\mathcal{F}$ com um número finito de singularidades $\left\{b_{j}^{1}\right\} \cup \ldots \cup\left\{b_{j}^{n}\right\}$. Finalmente, pelo lema 12, obtemos uma seção com apenas uma singularidade. 


\section{Capítulo 4}

\section{Campos de caminhos em variedades topológicas}

\subsection{Campos de caminhos com apenas uma singu- laridade}

Definição 15. Um espaço topológico $X$ é localmente homogêneo se, para todo $x \in X$, existem uma vizinhança $U$ de $x$ em $X$ e uma aplicação

$$
M: X \times U \times U \rightarrow X
$$

tal que

1. LH1: para todo par de pontos $a, b \in U$, a função $x \mapsto M(x, a, b), x \in X$, define um homeomorfismo de $X$ em si mesmo;

2. LH2: para todo $a, b \in U, M(a, a, b)=b$;

3. LH3: para todo a $\in U$ e todo $x \in X, M(x, a, a)=x$.

Definição 16. $\mathcal{F}=\left(E, E_{0}, p, B\right)$ é um par fibrado localmente trivial se $\mathcal{F}$ é um par fibrado com fibra $\left(F, F_{0}\right)$ e para todo $x \in B$, existe um aberto $U \subset B$ contendo $x$, e um homeomorfismo

$$
\psi_{U}:\left(U \times F, U \times F_{0}\right) \rightarrow\left(p^{-1}(U), p_{0}^{-1}(U)\right)
$$

tal que $p \circ \psi_{U}=\pi$, e consequentemente, $\pi \circ \psi_{U}^{-1}=p$ onde $\pi$ é a projeção na primeira coordenada. Em particular, temos que se $\mathcal{F}$ é um par fibrado localmente 
trivial, para todo $x \in B$, existe um aberto $U \subset B$ contendo $x$ tal que $\mathcal{F}_{\rfloor U}=$ $\left(p^{-1}(U), p_{0}^{-1}(U), p, U\right) \sim\left(U \times F, U \times F_{0}, \pi, U\right)$.

Antes de apresentarmos o principal resultado deste capítulo, segue um lema, adaptado de [11], que utilizaremos mais adiante.

Lema 13. Sejam $M$ uma n-variedade, $p: M \times M \rightarrow M$ a projeção na primeira coordenada, $\Delta=\left\{\left(x_{1}, x_{2}\right) \in M \times M \mid x_{1}=x_{2}\right\}$ e $\mathcal{M}=(M \times M, M \times M-\Delta, p, M)$. Então $\mathcal{M}$ é um par fibrado localmente trivial.

Demonstração. Sejam $h_{n}: \mathbb{R}^{n} \rightarrow V^{n}$, onde $V^{n}$ é o interior de $D^{n}$ o disco unitário de $\mathbb{R}^{n}$, o homeomorfismo dado por

$$
h_{n}(x)=\frac{x}{1+\|x\|}
$$

e a sua inversa $g_{n}: V^{n} \rightarrow \mathbb{R}^{n}$ dada por

$$
g_{n}(y)=\frac{y}{1-\|y\|}
$$

Obtemos uma aplicação $\gamma^{\prime}: V^{n} \times V^{n} \times D^{n} \rightarrow D^{n}$ dada por

$$
\gamma^{\prime}(y, z, w)= \begin{cases}h_{n}\left(g_{n}(w)+g_{n}(z)-g_{n}(y)\right), & w \in V^{n} \\ w & w \in \partial D^{n}\end{cases}
$$

$\gamma^{\prime}$ induz uma aplicação $\gamma^{\prime \prime}: V^{n} \times V^{n} \rightarrow G_{0}\left(D^{n}\right)$, onde $G_{0}\left(D^{n}\right)$ é o grupo de homomorfismos de $D^{n}$ que deixam $\partial D^{n}$ fixo pontualmente, $\gamma^{\prime \prime}$ satisfaz as seguintes propriedades:

(i) $\left[\gamma^{\prime \prime}(y, z)\right](y)=z, \quad(y, z) \in V^{n} \times V^{n}$;

(ii) $\gamma^{\prime \prime}(y, y)=1, \quad y \in V^{n}$;

(iii) $\gamma^{\prime \prime}(y, z)_{\rfloor \partial D^{n}}=1, \quad(y, z) \in V^{n} \times V^{n}$.

Dado $b \in M$, seja $U$ um aberto da n-variedade $M$ contendo $b$ e homeomorfo a $V^{n}$ cujo fecho é homemomorfo a $D^{n}$, compondo estes homeomorfismos com $\gamma^{\prime \prime}$ obtemos $\gamma: U \times U \rightarrow G(M)$, onde $G(M)$ é o grupo dos homomorfismos de $M, \gamma$ satisfaz as seguintes propriedades:

(i) $[\gamma(x, y)](x)=y, \quad(x, y) \in U \times U$;

(ii) $\gamma(x, x)=1, \quad x \in U$;

(iii) $\gamma(x, y)_{\downarrow M-U}=1, \quad(x, y) \in U \times U$. 
Seja

$$
\phi_{U}:(U \times M, U \times(M-b)) \rightarrow\left(p^{-1}(U), p_{0}^{-1}(U)\right)
$$

dada por $\phi_{U}(x, y)=(x,[\gamma(b, x)](y))$, como $\gamma$ é uma aplicação segue que $\phi_{U}$ é um homeomorfismo, com inversa dada por $\phi_{U}^{-1}(x, y)=\left(x,[\gamma(b, x)]^{-1}(y)\right)$. E como $p \circ$ $\phi_{U}=\pi$, segue que $\mathcal{M}$ é um par fibrado localmente trivial.

Observação: Na demonstração do lema 13 pudemos verificar que toda variedade topológica é um espaço localmente homogêneo.

Definição 17. Sejam B uma n-variedade, o conjunto

$$
T_{0}(B)=\left\{\alpha \in B^{I} \mid \alpha(t)=\alpha(0) \Leftrightarrow t=0\right\}
$$

é o espaço tangente total de $B$; se $b \in B$, o conjunto

$$
T_{0}(B, b)=\left\{\alpha \in B^{I} \mid \alpha(t)=b \Leftrightarrow t=0\right\}
$$

é o espaço tangente de $B$ em $b$. Sejam $T \subset B^{I}$ a união de $T_{0}$ com os caminhos constantes de $B$ e $q: T \rightarrow B$, dada por $q(\alpha)=\alpha(0)$. Um campo de caminhos $e ́$ uma seção em $(T, q, B)$, um campo de caminhos não singular é uma seção em $\left(T_{0}, q_{0}, B\right)$, onde $q_{0}=q_{\lrcorner T_{0}}$.

O lema seguinte é uma adaptação de [15].

Lema 14. Sejam $B$ um espaço topológico completamente regular, $b_{0} \in B$ e $U$ uma vizinhança de $b_{0}$ em $B$. Então existe uma homotopia $d_{t}:\left(T_{0}\left(B, b_{0}\right), T_{0}\left(U, b_{0}\right)\right) \rightarrow$ $\left(T_{0}\left(B, b_{0}\right), T_{0}\left(U, b_{0}\right)\right)$ tal que $d_{0}$ é a identidade e $d_{1}$ aplica $T_{0}\left(B, b_{0}\right)$ em $T_{0}\left(U, b_{0}\right)$.

Demonstração. Como $B$ é um espaço topológico completamente regular em $b_{0}$, existe uma função real contínua $\chi: B \rightarrow I$ tal que $\chi(X-U)=0$ e $\chi\left(b_{0}\right)=1$. Sejam $B^{*}=T_{0}\left(B, b_{0}\right)$ e $U^{*}=T_{0}\left(U, b_{0}\right)$. Seja $\phi: B^{*} \times I \rightarrow I$ a função contínua dada por

$$
\phi(\sigma, t)=\inf _{s \leq t} \chi[\sigma(s)]
$$

Fixado $\sigma \in B^{*}, \phi(\sigma, t)$ é uma função de $t$, não crescente com $\phi(\sigma, 0)=1$. Portanto, a equação $\phi(\sigma, t)=t$ possui uma única solução na variável $t$, dependendo continuamente de $\sigma$, denotemos por $\psi(\sigma)$ esta solução. Então, temos uma função contínua $\psi: B^{*} \rightarrow I$, como $\phi(\sigma, 0)=1 \neq 0$, temos que $\psi(\sigma)>0$ para todo $\sigma \in B^{*}$. A partir da aplicação $\psi$, podemos definir uma homotopia $d_{t}: B^{*} \rightarrow B^{*}$ que a cada $\sigma \in B^{*}$ 
associa o caminho

$$
d_{t}(\sigma): s \in I \mapsto \sigma[s-s t+s t \cdot \psi(\sigma)] .
$$

Notemos que $\psi(\sigma)>0 \Rightarrow 1-\psi(\sigma)<1 \Rightarrow t(1-\psi(\sigma))<1 \Rightarrow 1-t+t \psi(\sigma)>0$, ou seja, $\left[d_{t}(\sigma)\right](s)=\left[d_{t}(\sigma)\right](0) \Leftrightarrow s=0, \operatorname{logo}, d_{t}(\sigma) \in B^{*}$. Pela construção de $d_{t}$, segue que $d_{0}=i d_{B^{*}}$ e $d_{t}\left(U^{*}\right) \subset U^{*}$. Resta verficar apenas que $d_{1}$ leva $B^{*}$ em $U^{*}$. Para $\sigma \in B^{*}$ e $t \in I$ arbitrários, temos que

$$
\left[d_{1}(\sigma)\right](t)=\sigma(t \cdot \psi(\sigma))
$$

$t \cdot \psi(\sigma) \leq \psi(\sigma)$ e $\psi(\sigma)=\phi(\sigma, \psi(\sigma))=\inf _{s \leq \psi(\sigma)} \chi[\sigma(s)]$, logo,

$$
\chi[\sigma(t \cdot \psi(\sigma))] \geq \psi(\sigma)>0
$$

portanto, $\left[d_{1}(\sigma)\right](t) \in U^{*}$ e $d_{1}\left(B^{*}\right) \subset U^{*}$

Teorema 4. Toda n-variedade compacta admite um campo de caminhos com apenas uma singularidade.

Demonstração. Pelo teorema anterior, basta mostrar que $\mathcal{T}=\left(T, T_{0}, q, B\right)$ é um n-fvg. Primeiro, vamos mostrar que $(T, q, B)$ é um espaço fibrado. Como $B$ é uma variedade topológica, temos, pela observação acima, que $B$ é um espaço localmente homogêneo, e portanto, dado $b \in B$, existe uma vizinhança $U$ de $b$ em $B$, e uma aplicação

$$
\gamma: B \times U \times U \rightarrow B
$$

que satisfaz as propriedadas LH1, LH2 e LH3 da definição de espaço localmente homogêneo. Definamos uma aplicação

$$
\phi_{U}: U \times q^{-1}(U) \rightarrow T
$$

tal que $\phi_{U}(u, \sigma)$ é o caminho dado por

$$
\left[\phi_{U}(u, \sigma)\right](t)=\gamma(\sigma(t), \sigma(0), u)
$$

Por LH1, segue que $\phi_{U}(u, \sigma) \in T$, o que completa a construção de $\phi_{U}$. Por LH2, temos que

$$
q \circ \phi_{U}(u, \sigma)=\left[\phi_{U}(u, \sigma)\right](0)=\gamma(\sigma(0), \sigma(0), u)=u
$$


para todo $u \in U$ e todo $\sigma \in q^{-1}(U)$. Por LH3, temos que

$$
\left[\phi_{U}(u, \sigma)\right](t)=\gamma(\sigma(t), \sigma(0), u)=\sigma(t)
$$

se $u=q(\sigma)=\sigma(0)$, portanto, $\phi_{U}(q(\sigma), \sigma)=\sigma$. Segue, portanto, que $(T, q, B)$ satisfaz a PEF e, em particular, temos que $(T, q, B)$ é um espaço fibrado. A seção canônica do par fibrado $\left(T, T_{0}, q, B\right)$ é a função que a cada ponto de $B$ associa o caminho constante neste ponto, ou seja, $s: B \rightarrow T$ dada por $s(b)=\alpha$, onde $\alpha(t)=b$ para todo $t \in I$, e portanto, $T_{0}=T-s(B)$. Seja $\left(F, F_{0}\right)$ a fibra deste par, a contração de $F$ é obtida pela homotopia $h_{t}: F \rightarrow F$ dada por

$$
\left[h_{t}(\alpha)\right](s)=\alpha((1-t) \cdot s)
$$

onde $\alpha \in F$ e $s, t \in I$, portanto, $\mathcal{T}$ é um fvg. Sejam $U$ uma vizinhança euclidiana de $b_{0} \in B$, então $F \cap U^{I}=T\left(U, b_{0}\right)$ e $F_{0} \cap U^{I}=T_{0}\left(U, b_{0}\right)$. Então, pelo lema anterior, as inclusões $F \cap U^{I} \subset F$ e $F_{0} \cap U^{I} \subset F_{0}$ são equivalências homotópicas, logo, $\left(F \cap U^{I}, F_{0} \cap U^{I}\right) \sim\left(F, F_{0}\right)$. Seja $h: V \subset \mathbb{R}^{n} \rightarrow U$ um homeomorfismo tal que $h(0)=b_{0}$, isto é, $h:(V, V-0) \rightarrow\left(U, U-b_{0}\right)$. Sejam ainda $\beta:\left(F \cap U^{I}, F_{0} \cap U^{I}\right) \rightarrow$ $(U, U-0)$ dada por

$$
\beta(\omega)=\omega(1),
$$

e $\xi:\left(U, U-b_{0}\right) \rightarrow\left(F \cap U^{I}, F_{0} \cap U^{I}\right)$, que a cada $b \in U$ associa a imagem segundo $h$ do segmento que une $h^{-1}\left(b_{0}\right)$ a $h^{-1}(b)$,

$$
\xi(b)=h\left(\theta_{b}\right), \quad \theta_{b}(t)=h^{-1}\left(b_{0}\right)+t \cdot\left(h^{-1}(b)-h^{-1}\left(b_{0}\right)\right) .
$$

Então, segue que

$$
\beta \circ \xi(b)=\beta\left(h\left(\theta_{b}\right)\right)=\left[h\left(\theta_{b}\right)\right](1)=b,
$$

ou seja, $\beta \circ \xi=i d_{U}$. Temos ainda que,

$$
\xi \circ \beta(\omega)=\xi(\omega(1))=h\left(\theta_{\omega(1)}\right), \quad \theta_{\omega(1)}(t)=h^{-1}\left(b_{0}\right)+t \cdot\left(h^{-1}(\omega(1))-h^{-1}\left(b_{0}\right)\right) .
$$

Consideremos a homotopia $H_{s}: F \cap U^{I} \rightarrow F \cap U^{I}$ dada por

$$
\left[H_{s}(\omega)\right](t)= \begin{cases}\omega(t+2 s \cdot(1-t)), & 0 \leq s \leq 1 / 2 \\ h\left((2 s-1) \cdot(1-t) \cdot h^{-1}\left(b_{0}\right)+\right. & \\ \left.(1+(2 s-1) \cdot(t-1)) \cdot h^{-1}(\omega(1))\right), & 1 / 2 \leq s \leq 1\end{cases}
$$


Segue que $H_{0}=i d_{F \cap U^{I}}$ e $H_{1}=\xi \circ \beta$, ou seja, $\xi \circ \beta \sim i d_{F \cap U^{I}}$. Logo, $\left(F \cap U^{I}, F_{0} \cap U^{I}\right) \sim$ $\left(U, U-b_{0}\right)$ e como $h:(V, V-0) \rightarrow\left(U, U-b_{0}\right)$ é um homeomorfismo, segue que $\left(F \cap U^{I}, F_{0} \cap U^{I}\right) \sim(V, V-0)$. Finalmente, temos que $\left(F, F_{0}\right) \sim(V, V-0)$, ou seja, $F \sim \mathbb{R}^{n}$ e $F_{0} \sim S^{n-1}$, como $n \geq 2$ temos que $F_{0}$ é conexo por caminhos, e se $n \geq 3$ segue que $\pi_{1}\left(F_{0}\right)=0$, portanto, temos que $\mathcal{T}$ é um n-fvg.

Corolário 4. Seja B uma n-variedade compacta, então existe uma aplicação $f$ : $B \rightarrow B$ tal que $f$ é homotópica à identidade e $f$ possui no máximo um ponto fixo.

Demonstração. Pelo teorema anterior, $B$ admite um campo de caminhos $\sigma: M \rightarrow$ $T(B)$ com no máximo uma singularidade. Seja $f: M \rightarrow M$ a aplicação dada por

$$
f(y)=[\sigma(y)](1)
$$

A aplicação $f$ possui ponto fixo se, e somente se, $\sigma$ possui singularidades, ou seja, $f$ possui no máximo um ponto fixo. Seja $H_{t}: M \rightarrow M$ a homotopia dada por

$$
H_{t}(y)=[\sigma(y)](t)
$$

então $H_{0}(y)=[\sigma(y)](0)=y=i d(y)$ e $H_{1}(y)=[\sigma(y)](1)=f(y)$, e portanto, a aplicação $f$ é homotópica à identidade em $B$.

Definição 18. Seja $B$ uma n-variedade diferenciável. Um campo de vetores é uma seção no fibrado tangente de B. Um campo de vetores não singular é uma seção no fibrado de vetores tangentes não nulos.

Corolário 5. Toda n-variedade diferenciável compacta admite um campo de vetores com apenas uma singularidade.

\subsection{Número de Lefschetz e característica de Euler}

Sejam $\mathbb{K}$ um corpo e $V$ um $\mathbb{K}$-espaço vetorial finitamente gerado com base $e_{1}, \ldots, e_{n}$ e $\phi: V \rightarrow V$ um homomorfismo, definimos o traço de $\phi$ como sendo o número $\operatorname{tr} A$, onde $A$ é a matriz de $\phi$ nessa base. Se $X$ é um compacto ANR temos, pelo corolário da proposição 2 , que $H^{*}(X, \mathbb{K})$ é um espaço vetorial graduado tal que cada $H^{p}(X, \mathbb{K})$ é finitamente gerado e $H^{p}(X, \mathbb{K})$ é trivial exceto para um número finito de inteiros $p$ 's. Portanto, se $f: X \rightarrow X$ é uma aplicação, as seguintes somas estão bem definidas

$$
\operatorname{tr} f^{*}=\sum_{p} \operatorname{tr}\left(f_{p}^{*}\right)
$$


e a expressão que utilizaremos mais adiante

$$
\sum_{p}(-1)^{p} \operatorname{tr}\left(f_{p}^{*}\right)
$$

onde $f^{*}: H^{*}(X, \mathbb{K}) \rightarrow H^{*}(X, \mathbb{K})$ é o homomorfismo induzido pela $f$.

Definição 19. Sejam $X$ um compacto $A N R, f: X \rightarrow X$ uma aplicação e $\mathbb{K}$ um corpo, o número de Lefschetz de $f$ é dado por

$$
\Lambda_{f}(\mathbb{K})=\Lambda_{f}=\sum_{p}(-1)^{p} \operatorname{tr}\left(f_{p}^{*}\right)
$$

Definição 20. Seja $X$ um compacto $A N R$, a característica de Euler de $X$ é dada por

$$
\chi(X)=\Lambda_{i d_{X}}(\mathbb{Q})=\sum_{p}(-1)^{p} \operatorname{dim} H^{p}(X, \mathbb{Q}) .
$$

Lema 15. Sejam $X$ um compacto $A N R, f: X \rightarrow X$ uma aplicação, $P$ um poliedro que domina $X$ por meio das aplicações $\psi: P \rightarrow X$ e $\phi: X \rightarrow P$, e $g: P \rightarrow P$ a aplicação definida por $g=\phi \circ f \circ \psi$. Então $\Lambda_{f}(\mathbb{K})=\Lambda_{g}(\mathbb{K})$ para todo corpo $\mathbb{K}$.

A demonstração do resultado anterior pode ser encontrada em [4].

Proposição 19 (Teorema do ponto fixo de Lefschetz para compactos ANRs). Se $X$ é um compacto ANR e $f: X \rightarrow X$ uma aplicação tal que $\Lambda_{f}(\mathbb{K}) \neq 0$ para todo corpo $\mathbb{K}$, então toda aplicação homotópica a $f$ possui um ponto fixo.

Demonstração. Suponhamos que a aplicação $f$ não possui pontos fixos, vamos mostrar que neste caso temos que $\Lambda_{f}(\mathbb{K})=0$, para todo corpo $\mathbb{K}$. Como $X$ é um espaço métrico compacto, existe $\epsilon>0$ tal que, se $d: X \times X \rightarrow \mathbb{R}_{+}$é a métrica de $X$, $d(x, f(x))>\epsilon$ para todo $x \in X$. Seja $P$ o poliedro que, segundo a proposição 2 , $\epsilon$-domina $X$ por meio das aplicações $\psi: P \rightarrow X$ e $\phi: X \rightarrow P$. Vamos então mostrar que a aplicação $g=\phi \circ f \circ \psi: P \rightarrow P$ não possui ponto fixo. Suponhamos que existe $y \in P$ tal que $g(y)=y$, então

$$
\psi(y)=\psi g(y)=\psi \phi f \psi(y)
$$

Seja $h_{t}: X \rightarrow X$ a $\epsilon$-homotopia entre $\psi \circ \phi$ e $i d_{X}$, temos então que $\psi \phi f \psi(y)=$ 
$h_{0}(f \psi(y))$ e $f \psi(y)=h_{1}(f \psi(y)), \log$,

$$
\begin{aligned}
d(\psi(y), f \psi(y)) & =d(\psi \phi f \psi(y), f \psi(y)) \\
& =d\left(h_{0}(f \psi(y)), h_{1}(f \psi(y))\right) \\
& <\epsilon
\end{aligned}
$$

Seja $x=\psi(y) \in X$, então temos que existe $x \in X$ tal que $d(x, f(x))<\epsilon$, o que contradiz a definição de $\epsilon$. Portanto, $g$ não possui ponto fixo, e pelo teorema do ponto fixo de Lefschetz para poliedros, segue que $\Lambda_{g}(\mathbb{K})=0$, utilizando o lema anterior temos que $\Lambda_{f}(\mathbb{K})=\Lambda_{g}(\mathbb{K}), \operatorname{logo}, \Lambda_{f}(\mathbb{K})=0$

\section{3 Índice de ponto fixo}

No que se segue, sejam $M$ uma n-variedade, $f: M \rightarrow M$ uma aplicação com ponto fixo e $\mathcal{M}=(M \times M, M \times M-\Delta, p, M)$ o par fibrado do lema 13 .

Seja $x \in M, x$ é um ponto fixo isolado de $f$ se existe uma vizinhança $U$ de $x$ em $M$ tal que para todo $y \in U, f(y)=y$ se, e somente se, $y=x$.

Se $x$ é o único ponto fixo isolado de $f$ contido na vizinhança $U^{\prime}$ e $U^{\prime \prime}$ é uma vizinhança de $x$ tal que $\mathcal{M}_{\rfloor U^{\prime \prime}} \sim\left(U^{\prime \prime} \times M, U^{\prime \prime} \times(M-x)\right)$, seja $U=U^{\prime} \cap U^{\prime \prime}$, logo, pelo lema 13, existe um homeomorfismo $\phi$ tal que o seguinte diagrama comuta

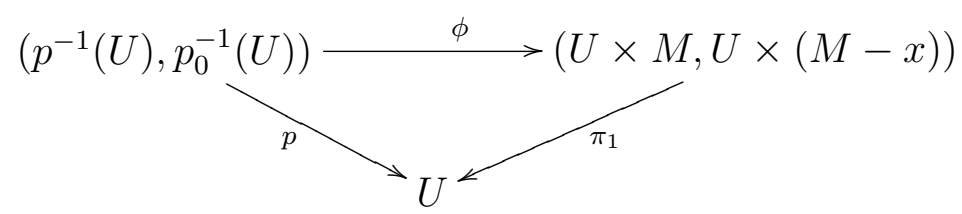

onde $\pi_{1}$ é a projeção na primeira coordenada.

A aplicação $f$ induz uma nova aplicação

$$
\begin{aligned}
(1 \times f): M & \rightarrow M \times M \\
y & \mapsto(y, f(y))
\end{aligned}
$$

e notemos que $(1 \times f)(U) \subset U \times M=p^{-1}(U)$, e

$$
y \in(U-x) \Rightarrow y \neq f(y) \Rightarrow(1 \times f)(y) \in[(U-x) \times M]-\Delta \subset(U \times M)-\Delta,
$$

ou seja, $(1 \times f)(U-x) \subset(U \times M)-\Delta \subset p_{0}^{-1}(U)$, e portanto,

$$
(1 \times f)(U, U-x) \subset\left(p^{-1}(U), p_{0}^{-1}(U)\right) .
$$


Consideremos ainda, a inclusão $i:(U, U-x) \rightarrow(M, M-x)$. Temos então, o seguinte diagrama

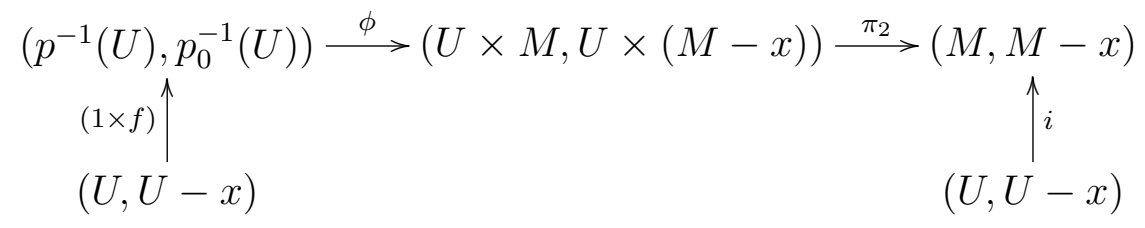

Consideremos os respectivos homomorfismos induzidos nos n-ésimos grupos de cohomologia singular. Por excisão, o homomorfismo induzido $i^{*}$ é um isomorfismo. E seja $F^{*}$ o homomorfismo definido pelo diagrama

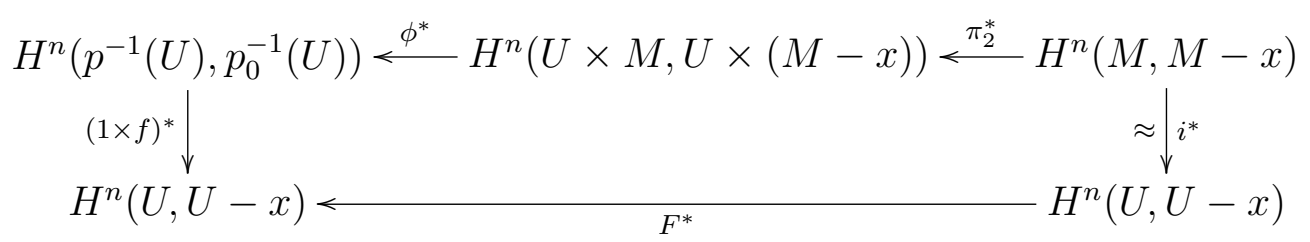

Sejam $\mu$ um gerador de $H^{n}(M, M-x) \approx \mathbb{Z}$ e $\mu_{U}=i^{*}(\mu)$, como $i^{*}$ é um isomorfismo temos que $H^{n}(U, U-x) \approx \mathbb{Z}$, e portanto, $F^{*}$ é da forma $F^{*}\left(\mu_{U}\right)=I \cdot \mu_{U}$, onde $I \in \mathbb{Z}$.

O índice do ponto fixo isolado $x$ da aplicação $f$ é o inteiro $I_{f}(x)$ tal que

$$
F^{*}\left(\mu_{U}\right)=I_{f}(x) \cdot \mu_{U}
$$

Proposição 20. Sejam $M$ uma n-variedade compacta, sem bordo e orientável, e $f: M \rightarrow M$ uma aplicação com pontos fixos $\left\{x_{1}, \ldots, x_{r}\right\}$, então

$$
\sum_{i=1}^{r} I_{f}\left(x_{i}\right)=(-1)^{n} \Lambda_{f}
$$

A demonstração do resultado anterior pode ser encontrada em [2].

Seja $f^{\prime}:(U, U-x) \rightarrow(M, M-x)$ dada por $f^{\prime}=\pi_{2} \circ \phi \circ(1 \times f)$

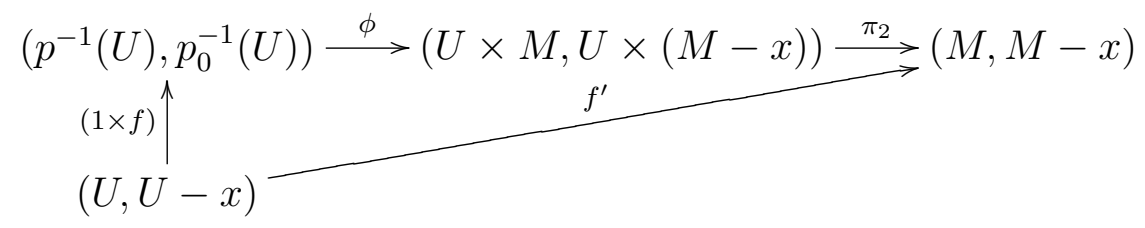

como $f^{\prime}$ é contínua, existe $U^{\prime} \subset M$ tal que $f\left(U^{\prime}\right) \subset U$, seja $V=U^{\prime} \cap U$, então $f^{\prime}(V) \subset f^{\prime}\left(U^{\prime}\right) \subset U, \log 0, f^{\prime}(V) \subset(U)$. Sejam $h:\left(E^{n}, E^{n}-0\right) \rightarrow(V, V-x)$ 
um homeomorfismo, $S=h\left(S^{n-1}\right) \subset(V-x)$ e $\bar{f}=f_{\rfloor_{S}}^{\prime}$, temos então que $\bar{f}(S)=$ $f_{\jmath S}^{\prime}(S) \subset f^{\prime}(V-x) \subset U-x$

Lema 16. Sejam $M$ uma n-variedade e $f: M \rightarrow M$ uma aplicação com um ponto fixo isolado $x$. Então $I_{f}(x)=0$ se, e somente se, $\bar{f}: S \rightarrow U-x$ é homotópica a uma aplicação constante.

Demonstração. Seja $j:(V, V-x) \rightarrow(U, U-x)$ a inclusão, como $f^{\prime}(V) \subset U$, podemos definir $f^{\prime \prime}=f^{\prime} \circ j:(V, V-x) \rightarrow(U, U-x)$, e temos o seguinte diagrama comutativo

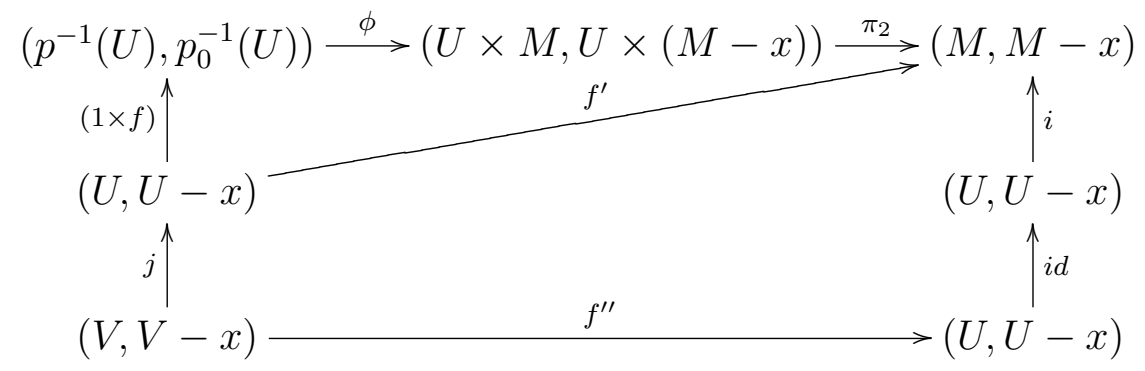

Sejam $k: S \rightarrow V-x$ a inclusão, $r: U-x \rightarrow S$ uma retração forte por deformação e $\bar{f}=f_{\rfloor S}^{\prime}: S \rightarrow U-x$, temos então o seguinte diagrama comutativo

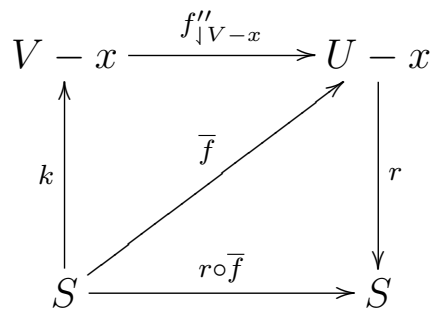

Para a inclusão $i:(U, U-x) \rightarrow(M, M-x)$ temos que $F^{*} i^{*}=f^{*}$, e para a inclusão $j:(V, V-x) \rightarrow(U, U-x)$ temos que $j^{*} F^{*} i^{*}=j^{*} f^{* *}=f^{\prime \prime *} i^{*}$. Da sequência exata de cohomologia de $(U, U-x)$ obtemos o isomorfismo natural em relação a funções contínuas

$$
\delta_{U}^{*}: H^{n-1}(U-x) \rightarrow H^{n}(U, U-x)
$$


de forma que o diagrama abaixo comuta

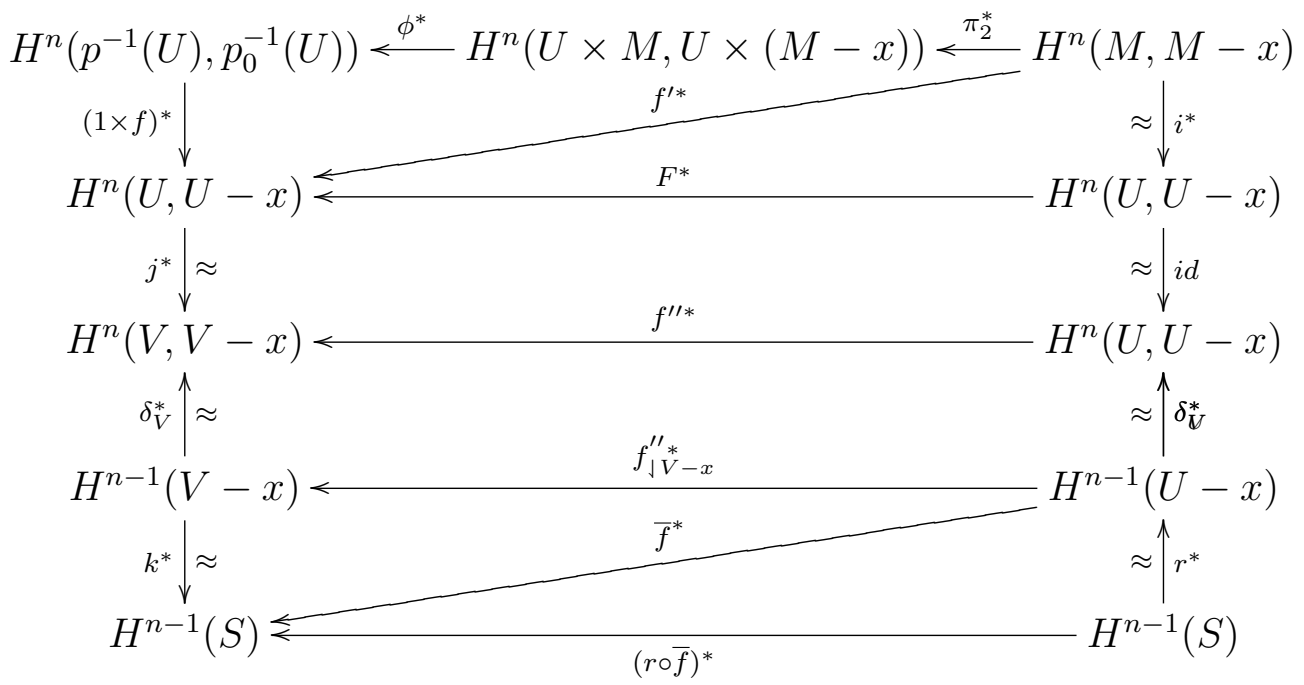

$(\Rightarrow)$ Se $I_{f}(x)=0$, então $F^{*}$ é o homomorfismo nulo, e portanto $(r \circ \bar{f})^{*}$ também é o homomorfismo nulo. Portanto, o grau de $r \circ \bar{f}: S \rightarrow S$ é zero, e $r \circ \bar{f}$ é homotópica a uma aplicação constante. Seja $k^{\prime}: S \rightarrow U-x$ a inclusão, então $k^{\prime} \circ r \circ \bar{f}: S \rightarrow U-x$ é homotópica a uma aplicação constante. Como $r$ é um retrato forte por deformação, temos que $k^{\prime} \circ r \sim i d_{U-x}$, e portanto, $k^{\prime} \circ r \circ \bar{f} \sim \bar{f}$ e $\bar{f}$ é homotópica a uma aplicação constante.

$(\Leftarrow)$ Se $\bar{f}$ é homotópica a uma aplicação constante, então $\bar{f}^{*}$ é o homomorfismo nulo, e portanto, $F^{*}$ também o é, $\operatorname{logo} I_{f}(x)=0$.

\subsection{Campos de caminhos sem singularidades}

Lema 17. Sejam $M$ uma n-variedade, $U$ uma vizinhança de $x$ em $M$,

$$
F_{0}^{\prime}=\left\{\alpha \in U^{I} \mid \alpha(t)=x \Leftrightarrow t=0\right\}
$$

e $\psi^{\prime}: F_{0}^{\prime} \rightarrow U-x$ dada por $\psi^{\prime}(\alpha)=\alpha(1)$, então $\psi^{\prime}$ é uma equivalência homotópica.

Demonstração. Sejam $h:\left(E^{n}, E^{n}-0\right) \rightarrow(U, U-x)$ um homeomorfismo e

$$
E_{0}=\left\{\alpha \in\left(E^{n}\right)^{I} \mid \alpha(t)=0 \Leftrightarrow t=0\right\}
$$

Então $h$ induz um homeomorfismo $\bar{h}: E_{0} \rightarrow F_{0}^{\prime}$ dado por

$$
[\bar{h}(\alpha)](t)=h(\alpha(t))
$$


Seja $\bar{\psi}: E_{0} \rightarrow E^{n}-0$ dado por

$$
\bar{\psi}(\alpha)=\alpha(1)
$$

O seguinte diagrama comuta

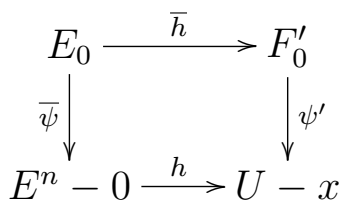

Se $\alpha \in E_{0}$, então $h^{-1} \circ \psi^{\prime} \circ \bar{h}(\alpha)=h^{-1}(h(\alpha(1)))=\alpha(1)=\bar{\psi}(\alpha)$. Portanto, basta mostrar que $\bar{\psi}$ é uma equivalência homotópica. Seja $\bar{\eta}:\left(E^{n}-0\right) \rightarrow E_{0}$ dada por

$$
[\bar{\eta}(y)](t)=t \cdot y
$$

então $\bar{\psi} \circ \bar{\eta}=[\bar{\eta}(y)](1)=1 \cdot y=i d_{E^{n}-0}(y)$, e $[\bar{\eta} \circ \bar{\psi}(\alpha)](t)=\alpha(1) \cdot t$. Consideremos a homotopia $H_{r}: E_{0} \rightarrow E_{0}$ dada por

$$
\left[H_{r}(\alpha)\right](t)= \begin{cases}\frac{t}{r} \cdot \alpha(r), & 0 \leq t<r \\ \alpha(t), & r \leq t \leq 1\end{cases}
$$

então $\left[H_{0}(\alpha)\right](t)=\alpha(t)=\left[i d_{E_{0}}(\alpha)\right](t)$ e $\left[H_{1}(\alpha)\right](t)=t \cdot \alpha(1)=[\bar{\eta} \circ \bar{\psi}(\alpha)](t)$, logo, $\bar{\eta} \circ \bar{\psi} \sim i d_{E_{0}}$, e portanto, $\bar{\psi}$ é uma equivalência homotópica.

Teorema 5. Uma n-variedade orientável compacta $M$ admite um campo de caminhos sem singularidades se, e somente se, $\chi(M)=0$.

Demonstração. $(\Rightarrow)$ Sejam $\sigma: M \rightarrow T_{0}$ um campo de caminhos sem singularidades, e $f: M \rightarrow M$ a aplicação dada por

$$
f(y)=[\sigma(y)](1)
$$

Como $\sigma$ não possui singularidades, temos que, para todo $y \in M$,

$$
[\sigma(y)](t)=y \Leftrightarrow t=0
$$

logo, a aplicação $f$ não possui pontos fixos, e pela proposição 20 , temos que $\Lambda_{f}=0$, onde $\Lambda_{f}$ é o número de Lefschetz de $f$. A aplicação $f$ é homotópica a identidade, então $\Lambda_{f}=\Lambda_{i d}=\chi(M)$. Portanto, $\chi(M)=0$. 
$(\Leftarrow)$ Suponhamos que $\chi(M)=0$, precisamos construir um campo de caminhos sem singularidades em $M$. Consideremos os pares fibrados $\mathcal{M}=(M \times M, M \times$ $M-\Delta, p, M), \mathcal{T}=\left(T, T_{0}, q, M\right)$. Pelo teorema 4, existe um campo de caminhos $\sigma^{\prime}: M \rightarrow T$ com uma singularidade, seja $x$ essa singularidade. Consideremos ainda

$$
\left(p^{-1}(x), p_{0}^{-1}(x)\right)=(M, M-x),\left(q^{-1}(x), q_{0}^{-1}(x)\right)=\left(F, F_{0}\right) .
$$

Pelo lema 13, existem $U$ uma vizinhança de $x$ em $M$ e um homeomorfismo $\phi$ tal que o diagrama abaixo comuta

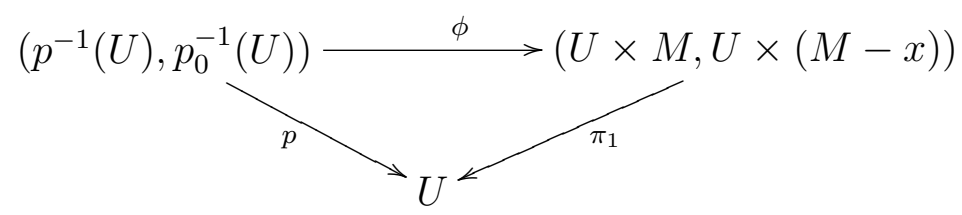

Seja $\phi^{\prime}:\left(q^{-1}(U), q_{0}^{-1}(U)\right) \rightarrow\left(U \times F, U \times F_{0}\right)$ o homeomorfismo dado por $\phi^{\prime}(\alpha)=$ $(u, \beta)$, onde

$$
\begin{gathered}
u=\pi_{1}(\phi(\alpha(0), \alpha(1))), \quad \mathrm{e} \\
\beta: t \in I \mapsto \pi_{2}(\phi(\alpha(0), \alpha(t))) \in M .
\end{gathered}
$$

Sejam

$$
\begin{aligned}
\psi: q^{-1}(U) & \rightarrow M \times M \\
\alpha & \mapsto(\alpha(0), \alpha(1)),
\end{aligned}
$$

e

$$
\begin{aligned}
\psi^{\prime}: F & \rightarrow M \\
\alpha & \mapsto \alpha(1) .
\end{aligned}
$$

Se $\alpha \in q^{-1}(U)$, então $\alpha(0) \in U$ e $\psi(\alpha)=(\alpha(0), \alpha(1)) \in U \times M$, e se $\alpha \in q_{0}^{-1}(U)$, então $\alpha(0) \in U, \alpha(0) \neq \alpha(1)$ e $\psi(\alpha)=(\alpha(0), \alpha(1)) \in(U \times M)-\Delta$, como vimos anteriormente, temos que $U \times M=p^{-1}(U)$ e $(U \times M)-\Delta=p_{0}^{-1}(U)$, logo, $\psi$ é uma aplicação de pares $\psi:\left(q^{-1}(U), q_{0}^{-1}(U)\right) \rightarrow\left(p_{0}^{-1}(U), p^{-1}(U)\right)$, e, como $\psi$ preserva fibras, temos que $\psi$ é uma aplicação de pares fibrados. De forma semelhante, temos que se $\alpha \in F_{0}$, então $\alpha(0) \neq \alpha(1) \Rightarrow x \neq \alpha(1)$ e $\psi^{\prime}(\alpha) \in M-x$, logo, $\psi^{\prime}$ é uma aplicação de pares $\psi^{\prime}:\left(F, F_{0}\right) \rightarrow(M, M-x)$. Temos, portanto, o seguinte diagrama

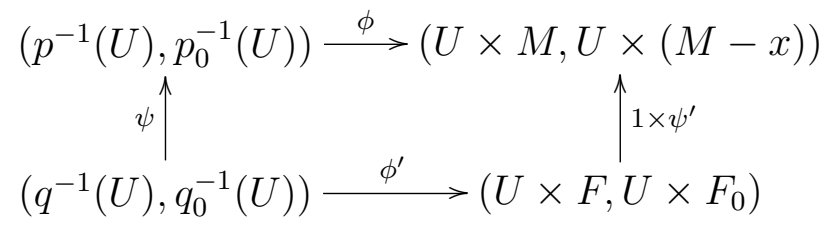


Para verificarmos que este diagrama comuta, tomemos $\alpha \in q^{-1}(U)$, então

$$
\psi(\alpha)=(\alpha(0), \alpha(1)) \Rightarrow \phi \circ \psi(\alpha)=\phi(\alpha(0), \alpha(1))
$$

e,

$$
\begin{aligned}
\phi^{\prime}(\alpha)=(u, \beta) \Rightarrow\left(1 \times \psi^{\prime}\right) \circ \phi^{\prime}(\alpha)=\left(1 \times \psi^{\prime}\right)(u, \beta)=(u, \beta(1)) & = \\
=\left(\pi_{1}(\phi(\alpha(0), \alpha(1))), \pi_{2}(\phi(\alpha(0), \alpha(1)))\right) & =\phi(\alpha(0), \alpha(1))
\end{aligned}
$$

Consideremos a aplicação

$$
\Sigma=\pi_{2} \circ \phi^{\prime} \circ \sigma^{\prime}:(U, U-x) \rightarrow\left(F, F_{0}\right) .
$$

Como $I$ é compacto e $U$ é um aberto, temos que $W(I, U)=\{f \in F \mid f(I) \subset U\}$ é um aberto de $F$. Como $\Sigma(x)$ é o caminho constante em $x$, e $x \in U$, temos que $\Sigma(x) \in W(I, U)$. E como $\Sigma$ é contínua, temos que existe $V$, uma vizinhança aberta de $x, V \subset U$, tal que para todo $y \in V, \Sigma(y) \in W(I, U)$, ou seja, $\Sigma(y) \in U^{I}$. Sejam $h:\left(E^{n}, E^{n}-0\right) \rightarrow(V, V-x)$ um homeomorfismo e $S=h\left(S^{n-1}\right)$, então como $S \subset V-x$ temos que $\Sigma_{\mid S}(S) \subset F_{0}^{\prime}=\left\{\alpha \in U^{I} \mid \alpha(t)=x \Leftrightarrow t=0\right\}$, seja $\bar{\sigma}=\Sigma_{\rfloor S}: S \rightarrow F_{0}^{\prime}$. Sejam $f: M \rightarrow M$ dada por

$$
f(y)=\left[\sigma^{\prime}(y)\right](1)
$$

e $\bar{f}: S \rightarrow M$ dada por

$$
\bar{f}=\left(\pi_{2} \circ \phi \circ(1 \times f)\right)_{\rfloor S} .
$$

Temos, portanto, o seguinte diagrama comutativo

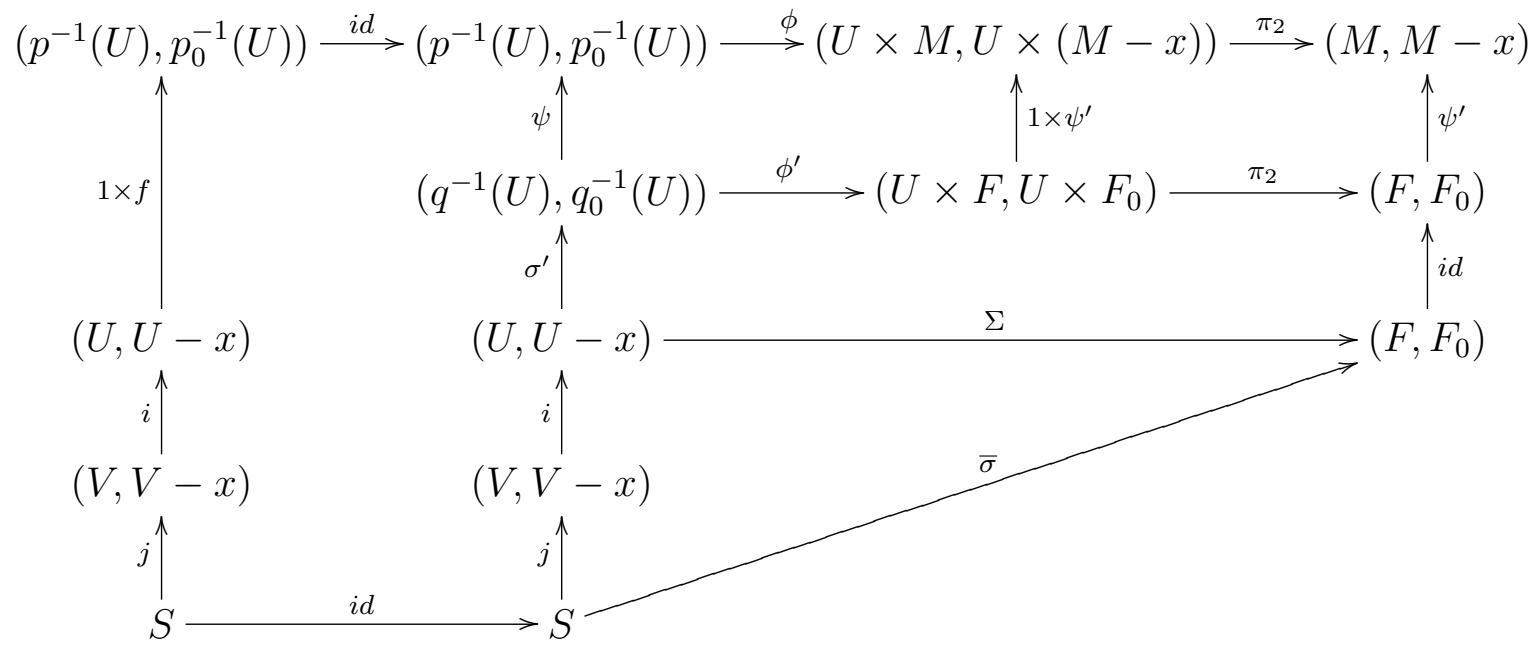


onde $i:(V, V-x) \rightarrow(U, U-x)$ e $j: S \rightarrow(V-x)$ são inclusões. Portanto, temos que

$$
\begin{aligned}
\psi^{\prime} \circ \bar{\sigma}=\psi^{\prime} \circ \pi_{2} \circ \phi^{\prime} \circ \sigma_{\lrcorner S}^{\prime}=\pi_{2} \circ(1 \times \psi) \circ \phi^{\prime} \circ \sigma_{\rfloor S}^{\prime} & = \\
& =\pi_{2} \circ \phi \circ \psi \circ \sigma_{\jmath_{S}}^{\prime}=\pi_{2} \circ \phi \circ(1 \times f)_{\rfloor S}=\bar{f}
\end{aligned}
$$

Como $\bar{\sigma}(S) \subset F_{0}^{\prime}$ e $\psi^{\prime}\left(F_{0}^{\prime}\right) \subset U-x$, segue que $\bar{f}(S) \subset U-x$. Consideremos a homotopia $H_{t}: M \rightarrow M$ dada por

$$
H_{t}(y)=\left[\sigma^{\prime}(y)\right](t)
$$

então $H_{0}(y)=\left[\sigma^{\prime}(y)\right](0)=y=i d(y)$ e $H_{1}(y)=\left[\sigma^{\prime}(y)\right](1)=f(y)$, logo, $f \sim 1$, e portanto, $\Lambda_{f}=\Lambda_{i d}$. Como $\Lambda_{i d}=\chi(M)$ e por hipótese $\chi(M)=0$, segue que $\Lambda_{f}=0$. Para todo $y \in M$ temos que $y=\left[\sigma^{\prime}(y)\right](0)$ e $f(y)=\left[\sigma^{\prime}(y)\right](1)$, logo, $y$ é ponto fixo de $f$ se, e somente se, $y$ é uma singularidade, e portanto, $x$ é o único ponto fixo de $f$. Como $M$ é uma n-variedade compacta e orientável, pela proposição 20, temos que $I_{f}(x)=(-1)^{n} \Lambda_{f}=0$. Portanto, pelo lema $16, \bar{f}: S \rightarrow U-x$ é homotópica a uma aplicação constante, e pelo lema anterior, temos que $\psi^{\prime}$ é uma equivalência homotópica, logo, $\bar{\sigma}: S \rightarrow F_{0}^{\prime}$ também é homotópica a uma aplicação constante. Consideremos novamente o homeomorfismo $h:\left(E^{n}, E^{n}-0\right) \rightarrow(V, V-x)$ e $B^{n}$ a bola unitária em $E^{n}$ centrada na origem. Se $C=h\left(B^{n}\right)$, então $S=\partial C$. Como $\bar{\sigma}: S \rightarrow F_{0}^{\prime}$ é homotópica a uma aplicação constante, podemos estendê-la a uma aplicação $\tau: C \rightarrow F_{0}^{\prime}$, e portanto, podemos construir $\sigma: M \rightarrow T_{0}$ dado por

$$
\sigma(y)= \begin{cases}\sigma^{\prime}(y), & y \in M-C \\ \phi^{\prime-1}(y, \tau(y)), & y \in C\end{cases}
$$

Notemos que, se $y \in C$, e lembrando que $C=h\left(B^{n}\right) \subset V \subset U$, temos que $\tau(y) \in$ $F_{0}^{\prime}, \log \mathrm{o},(y, \tau(y)) \in U \times F_{0}$, e como $\phi^{\prime}$ é um homeomorfismo, segue que $\sigma(y)=$ $\phi^{\prime-1}(y, \tau(y)) \in q_{0}^{-1}(U)$, e portanto, $\sigma$ não possui singularidades. Lembrando que o homeomorfismo $\phi^{\prime}$ é dado por $\phi^{\prime}(\alpha)=(u, \beta)$, onde

$$
\begin{gathered}
u=\pi_{1}(\phi(\alpha(0), \alpha(1))), \quad \mathrm{e} \\
\beta: t \in I \mapsto \pi_{2}(\phi(\alpha(0), \alpha(t))) \in M,
\end{gathered}
$$

e conforme o lema 13, o homeomorfismo $\phi$ é dado por $\phi(a, b)=\left(a,[\gamma(x, a)]^{-1}(b)\right)$, sendo $\gamma$ a aplicação considerada durante a demonstração do lema 13, portanto, segue que

$$
u=\pi_{1}(\phi(\alpha(0), \alpha(1)))=\pi_{1}\left(\alpha(0),[\gamma(x, \alpha(0))]^{-1}(\alpha(1))\right)=\alpha(0) \quad \mathrm{e}
$$


$\beta: t \in I \mapsto \pi_{2}(\phi(\alpha(0), \alpha(t)))=\pi_{2}\left(\alpha(0),[\gamma(x, \alpha(0))]^{-1}(\alpha(t))\right)=[\gamma(x, \alpha(0))]^{-1}(\alpha(t))$ Logo, se $y \in \partial C$, então

$$
\phi^{\prime} \circ \sigma^{\prime}(y)=\left(\pi_{1} \circ \phi^{\prime} \circ \sigma^{\prime}(y), \pi_{2} \circ \phi^{\prime} \circ \sigma^{\prime}(y)\right)=\left(\left[\sigma^{\prime}(y)\right](0), \Sigma(y)\right)
$$

e como $\partial C=S$, segue que

$$
\Sigma(y)=\bar{\sigma}(y)=\tau(y),
$$

e portanto,

$$
\phi^{\prime} \circ \sigma^{\prime}(y)=(y, \tau(y)) .
$$

Finalmente, temos que $\sigma^{\prime}(y)=\phi^{\prime-1}(y, \tau(y))$, e portanto, $\sigma$ é contínua, sendo assim, um campo de caminhos sem singularidades.

Corolário 6. Seja M uma n-variedade orientável compacta, então existe uma aplicação $f: M \rightarrow M$ sem pontos fixos e homotópica à identidade se, e somente se, $\chi(M)=0$.

Demonstração. $(\Rightarrow)$ Se $f$ é homotópica à identidade, temos que $\Lambda_{f}=\Lambda_{i d}$. Se $f$ : $M \rightarrow M$ não possui pontos fixos, então, pela proposição 20 , temos que $(-1)^{n} \Lambda_{f}=0$. Como $\chi(M)=\Lambda_{i d}$, temos que $\chi(M)=0$.

$(\Leftarrow)$ Se $\chi(M)=0$, então, pelo teorema anterior, $M$ admite um campo de caminhos sem singularidades $\sigma$. Seja $f: M \rightarrow M$ a aplicação dada por

$$
f(y)=[\sigma(y)](1)
$$

A aplicação $f$ possui ponto fixo se, e somente se, $\sigma$ possui singularidades, logo, $f$ não possui pontos fixos. Seja $H_{t}: M \rightarrow M$ a homotopia dada por

$$
H_{t}(y)=[\sigma(y)](t)
$$

então $H_{0}(y)=[\sigma(y)](0)=y=i d(y)$ e $H_{1}(y)=[\sigma(y)](1)=f(y)$, e portanto, a aplicação $f$ é homotópica à identidade.

Corolário 7. Uma variedade diferenciável orientável compacta admite um campo de vetores sem singularidades se, e somente se, $\chi(M)=0$. 


\section{Referências Bibliográficas}

[1] R. Brooks. Dominating polyhedra. Unpublished, 2003.

[2] R. Brown. On the lefschetz fixed point formula. American Journal of Mathematics, 87(1): 1-10, jan 1965.

[3] R. Brown. Path fields on manifolds. Transactions of the American Mathematical Society, 118: 180-191, jun 1965.

[4] R. Brown. The Lefschetz Fixed Point Theorem. Scott Foresman, 1971.

[5] M. L. Curtis. The covering homotopy theorem. Proceedings of the American Mathematical Society, 7(4): 682-684, aug 1956.

[6] R. Daverman. Decompositions of Manifolds. Academic Press, 1986.

[7] G. Allaud e E. Fadell. A fibre homotopy extension theorem. Transactions of the American Mathematical Society, 104(2): 239-251, aug 1962.

[8] W. Hurewicz e H. Wallman. Dimension Theory. Princeton Univ. Press, 1941.

[9] P. H. Doyle e J. G. Hocking. A decomposition theorem for n-dimensional manifolds. Proceedings of the American Mathematical Society, 13(3): 469-471, jun 1962.

[10] E. Fadell. On fiber spaces. Transactions of the American Mathematical Society, 90(1): 1-14, jan 1959.

[11] E. Fadell. Generalized normal bundles for locally-flat imbeddings. Transactions of the American Mathematical Society, 114(2): 488-513, feb 1965.

[12] O. Hanner. Some theorems on absolute neighborhood retracts. Arkiv för Matematik, pages 389-408, 1950.

[13] S-T. Hu. A theorem on homotopy extension. Doklady Akad. Nauk. S.S.S.R., 57: 231-234, 1947.

[14] S-T. Hu. Homotopy Theory. Academic Press, 1959.

[15] S-T. Hu. Fibrings of enveloping spaces. Proceedings of the London Mathematical Society, 11(3): 691-707, 1961. 
[16] W. Hurewicz. On the concept of fiber space. Proceedings of the National Academy of Science (U.S.A.), 41: 956-961, 1955.

[17] S. Kaplan. Homology properties of arbitrary subsets of euclidean spaces. Transactions of the American Mathematical Society, 62(1): 248-271, sep 1947.

[18] E. L. Lima. Elementos de Topologia Geral. IMPA, 1970.

[19] J. Munkres. Elements Of Algebraic Topology. Prentice Hall, 2 edition, 1999.

[20] J. Nash. A path space and the stiefel-whitney classes. Proceedings of the National Academy of Science (U.S.A.), 41: 320-321, 1955. 